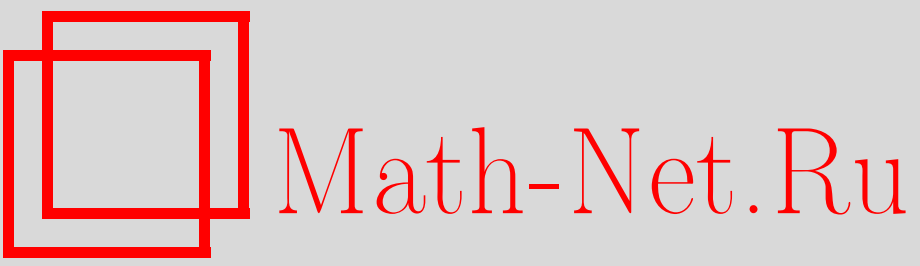

А. Ю. Колесов, Е. Ф. Мищенко, Н. Х. Розов, Явление буферности в резонансных системах нелинейных гиперболических уравнений, УМH, 2000, том 55, выпуск $2,95-120$

DOI: https://doi.org/10.4213/rm268

Использование Общероссийского математического портала Math-Net.Ru подразумевает, что вы прочитали и согласны с пользовательским соглашением

http://www . mathnet.ru/rus/agreement

Параметры загрузки:

IP: 52.23 .180 .231

26 апреля 2023 г., 15:01:45 


\title{
ЯВЛЕНИЕ БУФЕРНОСТИ В РЕЗОНАНСНЫХ СИСТЕМАХ НЕЛИНЕЙНЫХ ГИПЕРБОЛИЧЕСКИХ УРАВНЕНИЙ
}

\author{
А. Ю. Колесов, Е. Ф. Мищенко, Н. Х. РоЗОв
}

Исследуются гиперболические краевые задачи, представляющие собой системы телеграфных уравнений с нелинейными граничными условиями на концах конечного отрезка. Для данного класса систем устанавливается феномен буферности, т.е. существование в них при подходящем выборе параметров любого фиксированного числа устойчивых периодических по времени решений. Показано, что в случае резонансного спектра собственных частот изучение автоколебаний в различных системах приводит к одной из двух модельных краевых задач:

$$
\begin{aligned}
& \frac{\partial^{2} w}{\partial t \partial x}=w+\lambda\left(1-w^{2}\right) \frac{\partial w}{\partial x}, \quad w(t, x+1) \equiv-w(t, x), \quad \lambda>0 \\
& \frac{\partial w}{\partial t}+a^{2} \frac{\partial^{3} w}{\partial x^{3}}=w-w^{3}, \quad w(t, x+1) \equiv-w(t, x), \quad a \neq 0,
\end{aligned}
$$

являющихся своего рода инвариантами. Рассмотрены содержательные примеры из радиофизики.

Библиография: 29 названий.

\section{СОДЕРЖАНИЕ}

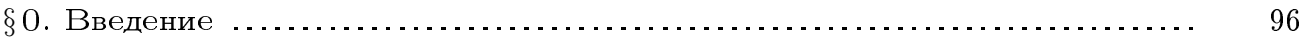

1. Разрушение буферности ...................................... 97

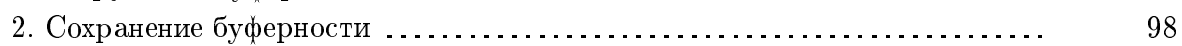

$\S 1$. Вывод базовых математических моделей ........................... 98

$\S 2$. Метод бесконечномерной нормализации . ........................... 101

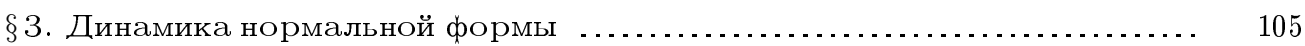

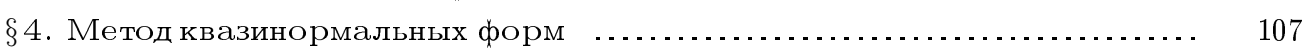

$\S 5$. Случай неэквидистантного спектра ........................... 111

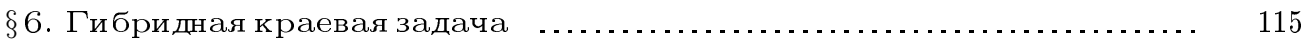

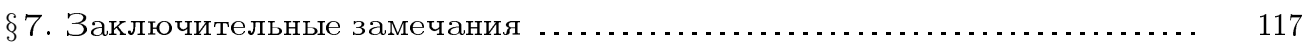

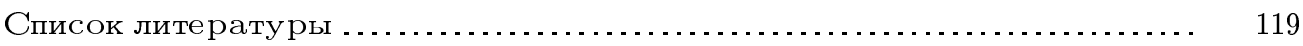

Работа выполнена при финансовой поддержке Российского фонда фундаментальных исследований (грант № 99-01-00157). 


\section{$\S$ 0. Введение}

Будем говорить о явлении буферности, если в некоторой системе дифференциальных уравнений с частными производными параболического или гиперболического типа при подходящем выборе параметров можно гарантировать существование любого фиксированного конечного числа устойчивых циклов (периодических по $t$ решений).

В системах параболических уравнений типа реакция-диффузия впервые это интересное явление численными методами было обнаружено Ю.С. Колесовым в ходе предпринятого по его инициативе в работе [1] исследования одной математической модели задачи хишник-жертва; рассматривавшаяся модель представляла собой краевую задачу для системы двух параболических уравнений с запаздыванием. Там же Ю. С. Колесов поставил проблему теоретического объяснения данного феномена на примере модельной параболической системы типа реакция-диффузия без запаздьвания. Эта проблема получила впоследствии свое решение в цикле публикаций [2]-[5]. И наконец, в статье [6] результаты [2]-[5] были перенесены непосредственно на исходную краевую задачу из [1].

Исследование буферности в краевых задачах гиперболического типа по инициативе Ю. С. Колесова начато в заметке [7]. Затем оно было продолжено в статьях [8]-[10], а свое логическое завершение получило в монографии [11]. Результаты этих работ показали, что буферность типична именно для гиперболических уравнений. Собственно говоря, и в параболических системах явление буферности обнаруживается лишь в тех случаях, когда они по своим свойствам в определенном смысле близки к гиперболическим системам. В гиперболических же системах возможна даже гипербуферность, т.е. сушествование сразу счетного числа устойчивых циклов (феномен гипербуферности подробно изучался в [10], [11]).

Проведенный в [7]-[11] математический анализ позволяет утверждать, что в первую очередь буферность характерна для широкого класса физических процессов, адекватные математические модели которых описываются гиперболическими уравнениями (например, для автоколебательных процессов в распределенных электрических и механических системах). В этой связи необходимо напомнить, что на принципиальную возможность существования сразу нескольких устойчивых циклов в классическом автогенераторе с распределенными параметрами (содержашем отрезок длинной линии) указьвал наш замечательньй физик А. А. Витт [12], [13] еше в начале 30-х годов. Однако в полном объеме феномен буферности как таковой в то время выявлен не был. По-видимому, впервые увеличение числа возможных (устойчивых) автоколебательных режимов при изменении параметров автогенератора удалось заметить экспериментально в работе, вьполненной под руководством В. В. Мигулина [14].

В настоящей статье объектами исследования являются краевые задачи гиперболического типа, состояшие из рассматриваемой на конечном отрезке (по пространственной переменной) системы телеграфных уравнений, чаще всего линейной. Нелинейности же фигурируют в граничных условиях на концах отрезка. Изучение подобных задач актуально в связи с тем, что они являются математическими моделями широкого класса автогенераторов, содержащих отрезок длинной линии с распределенньми индуктивностью, емкостью и сопротивлением. При этом нелинейные элементы, имеющие произвольную природу (классическая лампа, туннельньй диод и т.д.), в таких автогенераторах присоединены, как правило, к концам линии, что и объясняет происхождение нелинейных граничных условий. 
Предположим, что в задаче об устойчивости нулевого положения равновесия некоторой гиперболической системы из описанного выше класса реализуется критический случай счетного числа чисто мнимых собственных значений, а при изменении некоторых входящих в систему параметров происходит смещение части спектра устойчивости в правую комплексную полуплоскость. Тогда естественным образом возникает вопрос о сушествовании и устойчивости автоколебаний такой системы, бифурцирующих из нуля. Насколько нам известно, первой публикацией, посвященной данной проблеме, является уже упоминавшаяся вьше статья А. А. Витта [12]. Из ее результатов, а также из значительно более поздних работ [7]-[11] следует, что при отсутствии определенных резонансных соотношений между собственными частотами системы чаще всего в ней реализуется феномен буферности. Точнее говоря, в указанном случае имеется некоторое число квазигармонических (т.е. близких к гармоническим по $t)$ устойчивых циклов, и это число за счет подходящего выбора параметров может быть сделано сколь угодно большим. Кроме того, имеются еще неустойчивые квазигармонические инвариантные торы различных размерностей, разделяющие области притяжения разных устойчивых циклов.

Предположим теперь, что спектр собственных частот рассматриваемой гиперболической краевой задачи является эквидистантным (эквидистантность означает, что если занумеровать все собственные частоты в порядке возрастания, то расстояния между любыми двумя соседними из них будут одинаковы) или становится таковым при изменении некоторых параметров. В такой ситуации, которая весьма интересна в математическом плане и довольно часто встречается в приложениях, бифурцирующие из нуля автоколебания обладают некоторьми специфическими особенностями. При анализе ряда содержательных примеров (главным образом - из радиофизики) были обнаружены две следуюшие основные закономерности.

1. Разрушение буферности. Диапазон значений параметров, в котором реализуется феномен буферности, сушественно сужается и соответственно уменьшается количество устойчивых циклов. Более того, при прохождении некоторых параметров через конечные критические значения все циклы последовательно разрушаются за счет градиентных катастроф (т.е. становятся разрывными, оставаясь ограниченными в $C$-норме). Таким образом, динамика в данном случае характеризуется следующей схемой:

$$
\text { Буферность } \rightarrow \text { Градиентные катастрофы } \rightarrow \text { Разрьвные колебания } .
$$

Отметим, что схема (1) реализуется в двух основных ситуациях. Во-первых, она проявляется в наиболее часто встречаюшемся случае, когда в гиперболической системе с эквидистантным (или близким эквидистантному) спектром собственных частот увеличивается некий энергетический параметр, т.е. параметр, отвечающий за смещение спектра устойчивости в правую комплексную полуплоскость. При этом, как видно из (1), происходит переход от гладких колебаний к разрывным. Во-вторых, схема (1) может возникнуть при переходе в гиперболической системе от неэквидистантного спектра собственных частот к эквидистантному.

И наконец, что самое главное, в случае реализации схемы (1) исследование любой конкретной гиперболической системы приводит к одной и той же модельной краевой 
задаче [15]

$$
\frac{\partial^{2} w}{\partial t \partial x}=w+\lambda\left(1-w^{2}\right) \frac{\partial w}{\partial x}, \quad w(t, x+1) \equiv-w(t, x), \quad \lambda=\text { const }>0,
$$

или к некоторой незначительной ее модификации. Таким образом, задача (2) является своего рода инвариантом, характеризуюшим рассматриваемую особенность.

2. Сохранение буферности. Особенно интересно, что переход в гиперболической системе от неэквидистантного спектра к эквидистантному может вызвать и прямо противоположные последствия: увеличение количества сосуществующих устойчивых циклов, а также расширение диапазона значений параметров, в котором наблюдается само явление буферности. Специфика же резонансности здесь проявляется лишь в том, что часть устойчивых циклов теряет гармоническую форму. Подобная ситуация обнаруживается, например, при исследовании автоколебаний в длинной линии с туннельным диодом [16].

Отметим, наконец, что, как и в предыдушем случае, здесь также имеется инвариант - модельная краевая задача

$$
\frac{\partial w}{\partial t}+a^{2} \frac{\partial^{3} w}{\partial x^{3}}=w-w^{3}, \quad w(t, x+1) \equiv-w(t, x), \quad a=\text { const } \neq 0
$$

Настоящая работа подводит итог многолетнему циклу исследований феномена буферности в нелинейных гиперболических уравнениях. Ее цель - проиллюстрировать в очередной раз сформулированные выше общие положения на конкретном фактическом материале и получить, тем самым, дополнительные аргументы в пользу фундаментальности задач (2) и (3). В связи с этим ниже рассматриваются не вошедшие в монографию [11] радиофизические примеры - математические модели трех автогенераторов, представляющих собой распределенные аналоги классического генератора Ван-дер-Поля. На приводимых примерах описьвается физический вывод соответствуюших математических моделей, указывается способ получения из них задач (2) или (3), изучаются динамические свойства последних и демонстрируется используемьй математический аппарат - методы нормальных и квазинормальных форм, разработанные Ю. С. Колесовым [17], [18].

\section{§ 1. Вывод базовых математических моделей}

Изучение автогенераторов с распределенными параметрами актуально в связи с развитием микроэлектроники и с появившейся возможностью реализации автоколебательных систем на распределенных структурах. В частности, представляет интерес проблема создания автогенераторов с распределенными параметрами, “подобных" уже известным генераторам на сосредоточенных элементах. В настоящем параграфе, опираясь на идеи работ [19]-[21], опишем адекватные математические модели трех таких генераторов, представляющих собой распределенные аналоги "однолампового генератора Ван-дер-Поля с колебательным контуром в цепи сетки или цепи анода" [22].

Рассмотрим автогенератор, принципиальная схема которого показана на рис. 1a). Его характерная особенность в том, что имеются две линии, индуктивности $L_{j}$, емкости $C_{j}$ и активные сопротивления $R_{j}, j=1,2$, которых равномерно распределены 


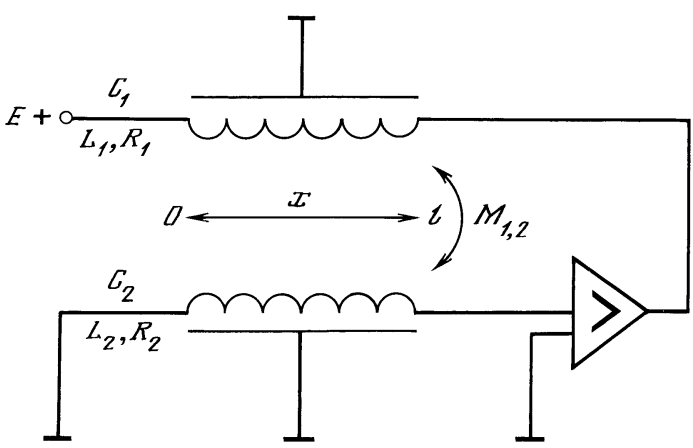

a)

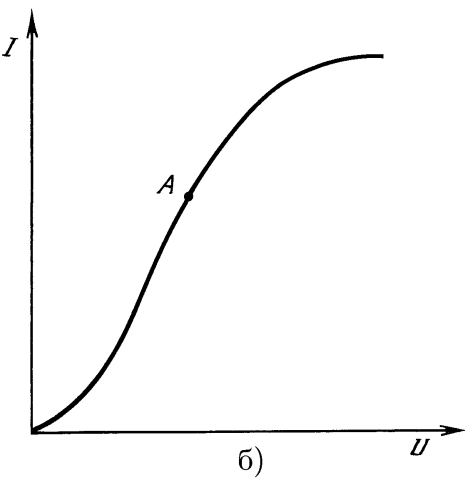

б)

Рис. 1

на некоторых отрезках проводников одной и той же длины $l$ (в обоих случаях пренебрегаем распределенной проводимостью). Считаем также, что между линиями наблюдается взаимоиндукция с коэффициентами $M_{j}, j=1,2$. Относительно нелинейного элемента (в традиционном случае - классической лампы) будем предполагать, что ток $I$ через него зависит от приложенного напряжения $u$ так, как показано на рис. 1б).

Обозначим через $u_{j}, i_{j}, j=1,2$, переменные составляющие напряжения и силы тока в линиях. Как известно, они связаны системой телеграфных уравнений

$$
\begin{array}{lll}
\frac{\partial u_{1}}{\partial x}=-R_{1} i_{1}-L_{1} \frac{\partial i_{1}}{\partial t}+M_{1} \frac{\partial i_{2}}{\partial t}, & \frac{\partial i_{1}}{\partial x}=-C_{1} \frac{\partial u_{1}}{\partial t}, \\
\frac{\partial u_{2}}{\partial x}=-R_{2} i_{2}-L_{2} \frac{\partial i_{2}}{\partial t}+M_{2} \frac{\partial i_{1}}{\partial t}, & \frac{\partial i_{2}}{\partial x}=-C_{2} \frac{\partial u_{2}}{\partial t} .
\end{array}
$$

В описьваемой ситуации ее следует рассматривать на отрезке $0 \leqslant x \leqslant l$, дополнив граничными условиями

$$
\left.u_{j}\right|_{x=0}=0, \quad j=1,2 ;\left.\quad i_{1}\right|_{x=l}=\left.\left[s_{0} u_{2}+s_{1} u_{2}^{2}-s_{2} u_{2}^{3}\right]\right|_{x=l},\left.\quad i_{2}\right|_{x=l}=0,
$$

где $s_{0}, s_{2}>0$, а знак $s_{1}$ произволен. Поясним происхождение граничных условий (1.2). $\mathrm{K}$ условию $\left.u_{1}\right|_{x=0}=0$ приходим в предположении, что источник постоянного напряжения $+E$ "шунтирован" достаточно большой емкостью и, следовательно, первая линия имеет на конце $x=0$ нулевое напряжение по переменной составляющей. Условие $\left.u_{2}\right|_{x=0}=0$ является следствием заземленности второй линии в точке $x=0$, a paвенство $\left.i_{2}\right|_{x=l}=0$ вытекает из предполагаемой идеальности усилителя (наличия у него бесконечно большого входного активного сопротивления). Наконец, фигурирующее в (1.2) нелинейное условие получается из связи двух рассматриваемых линий через нелинейньй элемент в предположении, что характеристика последнего аппроксимирована в окрестности рабочей точки $A$ (см. рис. 1б)) полиномом третьей степени с коэффишиентами $s_{j}, j=0,1,2$.

Краевая задача (1.1), (1.2) представляет собой общую математическую модель автогенератора, содержащего две $L C R$-линии, связанные через взаимоиндукцию и нелинейный элемент. Однако ниже будем изучать только два частных случая: $C_{2}=0$ или $C_{1}=0, R_{1}=0$. 
Начнем со случая $C_{2}=0$, когда задача $(1.1),(1.2)$ является моделью распределенного аналога классического генератора Ван-дер-Поля с колебательным контуром в цепи анода [22]. Для пояснения сути дела отметим, что первая линия играет здесь роль “колебательного контура в цепи анода", а вторая является вспомогательной, так как при $C_{2}=0$ автоматически имеем $i_{2} \equiv 0$ (ее функция состоит в создании - посредством взаимоиндукции - цепи обратной связи меж ду основной линией и усилителем).

Итак, исследованию подлежит краевая задача

$$
\begin{gathered}
\frac{\partial u_{1}}{\partial x}=-R_{1} i_{1}-L_{1} \frac{\partial i_{1}}{\partial t}, \quad \frac{\partial i_{1}}{\partial x}=-C_{1} \frac{\partial u_{1}}{\partial t}, \quad \frac{\partial u_{2}}{\partial x}=M_{2} \frac{\partial i_{1}}{\partial t} \\
\left.u_{1}\right|_{x=0}=\left.u_{2}\right|_{x=0}=0,\left.\quad i_{1}\right|_{x=l}=\left.\left[s_{0} u_{2}+s_{1} u_{2}^{2}-s_{2} u_{2}^{3}\right]\right|_{x=l}
\end{gathered}
$$

получаюшаяся из $(1.1),(1.2)$ при $i_{2}=0$. Для удобства ее анализа исключим сначала переменную $u_{2}$ :

$$
u_{2}=-\frac{M_{2}}{L_{1}} u_{1}-\frac{M_{2} R_{1}}{L_{1}} \int_{0}^{x} i_{1}(t, s) d s .
$$

Выполняя, далее, в $(1.3),(1.4)$ замены $x / l \rightarrow x, t /\left(l \sqrt{L_{1} C_{1}}\right) \rightarrow t, u_{1}=u$, $i_{1}=v \sqrt{C_{1} / L_{1}}$, убеждаемся, что математическая модель принимает вид

$$
\begin{array}{ll}
u_{t}=-v_{x}, & v_{t}=-u_{x}-\varepsilon v \\
\left.u\right|_{x=0}=0, & \left.v\right|_{x=1}+\beta_{0} \sigma+\beta_{1} \sigma^{2}-\beta_{2} \sigma^{3}=0
\end{array}
$$

где

$$
\begin{gathered}
\sigma=\left.u\right|_{x=1}+\varepsilon \int_{0}^{1} v(t, x) d x, \quad \varepsilon=R_{1} l \sqrt{C_{1} / L_{1}}, \quad \beta_{0}=\alpha s_{0} \sqrt{L_{1} / C_{1}}, \\
\beta_{1}=-\alpha^{2} s_{1} \sqrt{L_{1} / C_{1}}, \quad \beta_{2}=\alpha^{3} s_{2} \sqrt{L_{1} / C_{1}}, \quad \alpha=M_{2} / L_{1} .
\end{gathered}
$$

В случае $C_{1}=0, R_{1}=0$ имеем $i_{1} \equiv I(t)$, где $I(t)=\left.\left[s_{0} u_{2}+s_{1} u_{2}^{2}-s_{2} u_{2}^{3}\right]\right|_{x=l}$. Таким образом, здесь вместо (1.1), (1.2) рассмотрению подлежит краевая задача

$$
\begin{gathered}
\frac{\partial u_{2}}{\partial x}=-R_{2} i_{2}-L_{2} \frac{\partial i_{2}}{\partial t}+M_{2} \frac{d I}{d t}, \quad \frac{\partial i_{2}}{\partial x}=-C_{2} \frac{\partial u_{2}}{\partial t}, \\
\left.u_{2}\right|_{x=0}=0,\left.\quad i_{2}\right|_{x=l}=0,
\end{gathered}
$$

представляющая модель распределенного аналога генератора Ван-дер-Поля с колебательным контуром в цепи сетки [22]. Действительно, теперь вспомогательной является уже первая линия: в силу условия $R_{1}=0$ можно пренебречь падением напряжения в ней и считать, что $i_{1}=I(t)$. Отметим еще, что роль вспомогательной линии здесь та же, что и в классическом генераторе Ван-дер-Поля: через нее посредством взаимоиндукции осушествляется "подкачка" энергии в основную $L_{2} C_{2} R_{2}$-линию, пополняющая часть энергии последней, теряемой на $R_{2}$. Выполняя в задаче (1.6) замены $x / l \rightarrow x, t /\left(l \sqrt{L_{2} C_{2}}\right) \rightarrow t, u_{2}=u, i_{2}-M_{2} I(t) / L_{2}=v \sqrt{C_{2} / L_{2}}$, приведем ее к более удобному для дальнейшего анализа виду

$$
\begin{array}{ll}
u_{t}=-v_{x}, & v_{t}=-u_{x}-\varepsilon v-\left.\varepsilon\left[\beta_{0} u+\beta_{1} u^{2}-\beta_{2} u^{3}\right]\right|_{x=1}, \\
\left.u\right|_{x=0}=0,\left.\quad v\right|_{x=1}+\left.\beta_{0} u\right|_{x=1}+\left.\beta_{1} u^{2}\right|_{x=1}-\left.\beta_{2} u^{3}\right|_{x=1}=0
\end{array}
$$


где $\varepsilon=R_{2} l \sqrt{C_{2} / L_{2}}, \beta_{j}=s_{j} M_{2} / \sqrt{L_{2} C_{2}}, j=0,1,2$.

И наконец, рассмотрим еще один распределенный аналог генератора Ван-дер-Поля, получаюшийся из предыдушего при условии, что конец $x=0$ основной линии не заземлен, а разомкнут. В этом случае вместо (1.7) имеем дело с краевой задачей

$$
\begin{array}{ll}
u_{t}=-v_{x}, & v_{t}=-u_{x}-\varepsilon v-\left.\varepsilon\left[\beta_{0} u+\beta_{1} u^{2}-\beta_{2} u^{3}\right]\right|_{x=1}, \\
\left.v\right|_{x=0}=\left.v\right|_{x=1}, & \left.v\right|_{x=1}+\left.\beta_{0} u\right|_{x=1}+\left.\beta_{1} u^{2}\right|_{x=1}-\left.\beta_{2} u^{3}\right|_{x=1}=0 .
\end{array}
$$

В качестве фазовых пространств (пространств начальных условий $(u(x), v(x))$ ) краевых задач $(1.5),(1.7),(1.8)$ возьмем соответственно нелинейные многообразия $\Sigma_{j}$, $j=1,2,3$, в гильбертовом пространстве $W_{2}^{1}\left([0,1] ; \mathbb{R}^{2}\right)$, определяющиеся равенствами

$$
\begin{aligned}
& \Sigma_{1}=\left\{(u(x), v(x)):\left.u\right|_{x=0}=0,\left.v\right|_{x=1}+\beta_{0} \sigma+\beta_{1} \sigma^{2}-\beta_{2} \sigma^{3}=0\right\}, \\
& \Sigma_{2}=\left\{(u(x), v(x)):\left.u\right|_{x=0}=0,\left.v\right|_{x=1}+\left.\beta_{0} u\right|_{x=1}+\left.\beta_{1} u^{2}\right|_{x=1}-\left.\beta_{2} u^{3}\right|_{x=1}=0\right\}, \\
& \Sigma_{3}=\left\{(u(x), v(x)):\left.v\right|_{x=0}=\left.v\right|_{x=1}=-\left.\left[\beta_{0} u+\beta_{1} u^{2}-\beta_{2} u^{3}\right]\right|_{x=1}, \int_{0}^{1} u(x) d x=0\right\} .
\end{aligned}
$$

Напомним, что понятие фазового пространства позволяет обычным образом определять основные понятия математической теории колебаний. Отметим также, что однозначная разрешимость отвечаюших задачам (1.5), (1.7), (1.8) смешанных задач с начальными условиями из $\Sigma_{j}, j=1,2,3$, устанавливается стандартно: достаточно системы из (1.5), (1.7), (1.8) проинтегрировать по характеристикам, а затем результат подставить в указанные там же граничные условия (см. [11]). Заметим, наконец, что требование $\int_{0}^{1} u d x=0$ в определении $\Sigma_{3}$ вызвано равенством $\int_{0}^{1} u(t, x) d x \equiv \mathrm{const}$, справедливым для любого решения задачи (1.8).

\section{$\S$ 2. Метод бесконечномерной нормализации}

Периодическими по $t$ решениями краевых задач $(1.5),(1.7)$ мы будем интересоваться при дополнительных предположениях

$$
\beta_{0}=\varepsilon / 2+\gamma \varepsilon^{2}, \quad \beta_{1}=0, \quad \beta_{2}=1,
$$

где $0<\varepsilon \ll 1$, а параметр $\gamma>0$ имеет порядок единищы. Первое из условий $(2.1)$ продиктовано чисто математическими причинами, которые прояснятся ниже. Допущение $\beta_{1}=0$, означаюшее симметричность нелинейной характеристики, сделано лишш для простоты, ибо все получающиеся результаты сохраняются и при $\beta_{1} \neq 0$. Наконец, третье из равенств (2.1) легко обеспечивается подходящей нормировкой переменных $u, v$.

Подставляя (2.1) в задачу (1.5) и сохраняя во втором граничном условии (1.5) только сушественные для дальнейшего слагаемые, приходим к краевой задаче

$$
\begin{aligned}
& u_{t}=-v_{x}, \quad v_{t}=-u_{x}-\varepsilon v \\
& \left.u\right|_{x=0}=0,\left.\quad v\right|_{x=1}+\left.\left(\frac{\varepsilon}{2}+\gamma \varepsilon^{2}\right) u\right|_{x=1}+\frac{\varepsilon^{2}}{2} \int_{0}^{1} v d x-\left.u^{3}\right|_{x=1}=0 .
\end{aligned}
$$


Для построения ее периодических по $t$ решений воспользуемся методом бесконечномерной нормализации. Этот метод представляет собой специальный вариант асимптотического метода Крылова-Боголюбова-Митропольского [23] и смькается в алгоритмическом плане с методом квазинормальных форм Ю. С. Колесова [17], [18]. Следуя методике этих работ, подставим в (2.2) ряды

$$
\begin{aligned}
& u=\varepsilon u_{0}(t, \tau, x)+\varepsilon^{2} u_{1}(t, \tau, x)+\varepsilon^{3} u_{2}(t, \tau, x)+\cdots \\
& v=\varepsilon v_{0}(t, \tau, x)+\varepsilon^{2} v_{1}(t, \tau, x)+\varepsilon^{3} v_{2}(t, \tau, x)+\cdots
\end{aligned}
$$

где $\tau=\varepsilon^{2} t$, все функции являются 4-периодическими по $t$,

$$
\begin{aligned}
& u_{0}=\sum_{n=1}^{\infty}\left[z_{n}(\tau) \exp \left(i \omega_{n} t\right)+\bar{z}_{n}(\tau) \exp \left(-i \omega_{n} t\right)\right](-1)^{n-1} \sin \omega_{n} x \\
& v_{0}=i \sum_{n=1}^{\infty}\left[z_{n}(\tau) \exp \left(i \omega_{n} t\right)-\bar{z}_{n}(\tau) \exp \left(-i \omega_{n} t\right)\right](-1)^{n-1} \cos \omega_{n} x
\end{aligned}
$$

$\omega_{n}=\pi(2 n-1) / 2$, а комплексные “амплитуды" $z_{n}$ таковы, что сходится ряд с общим членом $\omega_{n}^{2}\left|z_{n}\right|^{2}$ (тогда $u_{0}, v_{0} \in W_{2}^{1}$ по переменной $x$ ). Приравнивая, далее, коэффиициенты при одинаковых степенях $\varepsilon$, последовательно определяем $u_{j}, v_{j}, j=1,2$, и неизвестные амплитуды $z_{n}, n=1,2, \ldots$ Подчеркнем, что отыскание автоколебаний краевой задачи (2.2) в виде (2.3) вполне естественно, так как соотношения (2.4) (при фиксированном $\tau$ ) задают произвольное решение линейной краевой задачи, получающейся из задачи (2.2) при $\varepsilon=0$ и после отбрасьвания нелинейности.

На первом шаге описанного алгоритма приходим к краевой задаче

$$
\frac{\partial u_{1}}{\partial t}=-\frac{\partial v_{1}}{\partial x}, \quad \frac{\partial v_{1}}{\partial t}=-\frac{\partial u_{1}}{\partial x}-v_{0},\left.\quad u_{1}\right|_{x=0}=0,\left.\quad v_{1}\right|_{x=1}+\left.\frac{1}{2} u_{0}\right|_{x=1}=0
$$

решение которой будем искать в виде рядов той же структуры, что и соответствующие неоднородности, т.е. в виде

$$
\begin{aligned}
& u_{1}=\sum_{n=1}^{\infty}\left(z_{n} A_{n}(x) \exp \left(i \omega_{n} t\right)+\bar{z}_{n} \bar{A}_{n}(x) \exp \left(-i \omega_{n} t\right)\right), \\
& v_{1}=\sum_{n=1}^{\infty}\left(z_{n} B_{n}(x) \exp \left(i \omega_{n} t\right)+\bar{z}_{n} \bar{B}_{n}(x) \exp \left(-i \omega_{n} t\right)\right) .
\end{aligned}
$$

На этом пути для $A_{n}, B_{n}$ получаем линейные неоднородные краевые задачи, разрешимость которых обеспечивает слагаемое $\left.(\varepsilon / 2) u\right|_{x=1}$ во втором граничном условии (2.2). Таким образом, теперь становятся понятными мотивы специального предположения в (2.1) относительно вида коэффициента усиления $\beta_{0}$. Несложньй анализ упомянутых краевых задач приводит к равенствам

$$
A_{n}=-\frac{i}{2}(-1)^{n-1} x \cos \omega_{n} x, B_{n}=\frac{i}{\omega_{n}}\left[A_{n}^{\prime}(x)+i(-1)^{n-1} \cos \omega_{n} x\right], \quad n=1,2, \ldots
$$


На втором шаге после исключения $v_{2}$ имеем дело с краевой задачей

$$
\begin{gathered}
\left(\frac{\partial^{2}}{\partial t^{2}}-\frac{\partial^{2}}{\partial x^{2}}\right) u_{2}=-2 \frac{\partial^{2} u_{0}}{\partial t \partial \tau}-\frac{\partial u_{1}}{\partial t}, \\
\left.u_{2}\right|_{x=0}=0,\left.\quad \frac{\partial u_{2}}{\partial x}\right|_{x=1}=\frac{d}{d t}\left[\left.\gamma u_{0}\right|_{x=1}-\left.u_{0}^{3}\right|_{x=1}\right],
\end{gathered}
$$

решение которой ишем в виде

$$
u_{2}=\sum_{n=1}^{\infty}\left(C_{n}(x, \tau) \exp \left(i \omega_{n} t\right)+\bar{C}_{n}(x, \tau) \exp \left(-i \omega_{n} t\right)\right) .
$$

Нетрудно проверить, что, с учетом $(2.7)$, для $C_{n}, n=1,2, \ldots$, получаются краевые задачи

$$
\begin{gathered}
\frac{d^{2} C_{n}}{d x^{2}}+\omega_{n}^{2} C_{n}=(-1)^{n-1}\left[2 i \omega_{n} \frac{d z_{n}}{d \tau} \sin \omega_{n} x+\frac{\omega_{n}}{2} z_{n} x \cos \omega_{n} x\right] \\
\left.C_{n}\right|_{x=0}=0,\left.\quad \frac{d C_{n}}{d x}\right|_{x=1}=i \omega_{n} f_{n} .
\end{gathered}
$$

Здесь $f_{n}$ - коэффициент при $\exp \left(i \omega_{n} y\right)$ ряда Фурье функции $\gamma w(\tau, y)-w^{3}(\tau, y)$, где

$$
w(\tau, y)=\sum_{n=1}^{\infty}\left(z_{n}(\tau) \exp \left(i \omega_{n} y\right)+\bar{z}_{n}(\tau) \exp \left(-i \omega_{n} y\right)\right) .
$$

Условия разрешимости задач (2.10) приводят к счетной системе обыкновенных дифференциальных уравнений

$$
\frac{d z_{n}}{d \tau}=\frac{i}{8 \omega_{n}} z_{n}+f_{n}, \quad n=1,2, \ldots,
$$

для отыскания неизвестных амплитуд $z_{n}, n=1,2, \ldots$ Привлекая, далее, функцию (2.11), без труда убеждаемся, что счетная система (2.12) "сворачивается" в краевую задачу

$$
\frac{\partial^{2} w}{\partial \tau \partial y}=-\frac{1}{8} w+\left[\gamma-3 w^{2}\right] \frac{\partial w}{\partial y}, \quad w(\tau, y+2) \equiv-w(\tau, y) .
$$

Строгий смысл изложенным формальньм построениям придается по той же схеме, что и в случае обыкновенных дифференциальных уравнений. Именно, с помошью построенных отрезков рядов (2.3) конструируется замена переменных, приводяшая исходную задачу (2.2), с точностью до слагаемых порядка $\varepsilon$, к виду (2.12) (см. [11]). Таким образом, система (2.12), а значит, и краевая задача (2.13) представляют собой "укороченную" нормальную форму задачи (2.2). Поэтому справедливо следуюшее стандартное утверждение о соответствии между их периодическими решениями.

Теорема 2.1. Пусть краевая задача (2.13) имеет периодическое решение $w=$ $w_{0}(\xi), \xi=\sigma_{0} \tau+y, \sigma_{0}=$ const, әкспоненциально орбитально устойчивое или 
дихотомичное (в метрике $W_{2}^{1}$ ). Тогда найдется такое $\varepsilon_{0}>0$, что при всех $0<\varepsilon \leqslant \varepsilon_{0}$ в краевой задаче (2.2) ему отвечает иикл (периодическое по $t$ решение) той эсе устойчивости с асимптотикой (2.3). При этом в формулах (2.4) следует полохить

$$
z_{n}(\tau)=w_{n}^{0} \exp \left(i \omega_{n} \sigma_{0} \tau\right), \quad n=1,2, \ldots
$$

где $w_{n}^{0}, \bar{w}_{n}^{0}-$ коэффициенть Фурье функции $w_{0}(\xi)$ по системе $\exp \left( \pm i \omega_{n} \xi\right), n \geqslant 1$.

Обратимся теперь к краевой задаче (1.7). Подставляя в нее $(2.1),(2.3),(2.4)$ и действуя описанньм вьше образом, снова приходим к краевой задаче (2.13). Поэтому аналог теоремы 2.1 здесь также остается в силе.

В случае краевой задачи (1.8) возникает ряд новых моментов. Начнем с того, что, в отличие от двух предыдуших случаев, здесь автоколебания будем строить при условиях

$$
\beta_{0}=\varepsilon / 4+\gamma \varepsilon^{2}, \quad \beta_{1}=0, \quad \beta_{2}=1
$$

где по-прежнему $0<\varepsilon \ll 1$, а параметр $\gamma>0$ имеет порядок единишы.

Подставим, далее, в задачу (1.8) равенства (2.14) и ряды (2.3), в которых теперь

$$
\begin{aligned}
& u_{0}=\sum_{n=1}^{\infty}\left[z_{n}(\tau) \exp \left(i \omega_{n} t\right)+\bar{z}_{n}(\tau) \exp \left(-i \omega_{n} t\right)\right] \cos \omega_{n} x \\
& v_{0}=-i \sum_{n=1}^{\infty}\left[z_{n}(\tau) \exp \left(i \omega_{n} t\right)-\bar{z}_{n}(\tau) \exp \left(-i \omega_{n} t\right)\right] \sin \omega_{n} x
\end{aligned}
$$

$\omega_{n}=\pi(2 n-1), n=1,2, \ldots$, и сходится ряд с общим членом $\omega_{n}^{2}\left|z_{n}\right|^{2}$. В результате после приравнивания коэффициентов при $\varepsilon^{2}$ приходим к аналогичной (2.5) краевой задаче

$$
\frac{\partial u_{1}}{\partial t}=-\frac{\partial v_{1}}{\partial x}, \quad \frac{\partial v_{1}}{\partial t}=-\frac{\partial u_{1}}{\partial x}-v_{0},\left.\quad v_{1}\right|_{x=0}=\left.v_{1}\right|_{x=1}=-\left.\frac{1}{4} u_{0}\right|_{x=1},
$$

решение которой также будем искать в виде рядов (2.6). В итоге для $A_{n}, B_{n}$ получаем равенства

$$
A_{n}=\frac{i}{2}\left(x-\frac{1}{2}\right) \sin \omega_{n} x, \quad B_{n}=\frac{i}{\omega_{n}}\left[A_{n}^{\prime}(x)-i \sin \omega_{n} x\right], \quad n=1,2, \ldots
$$

На втором шаге после исключения $v_{2}$ имеем дело с аналогичной (2.8) краевой задачей

$$
\begin{gathered}
\left(\frac{\partial^{2}}{\partial t^{2}}-\frac{\partial^{2}}{\partial x^{2}}\right) u_{3}=-\frac{\partial u_{1}}{\partial t}-2 \frac{\partial^{2} u_{0}}{\partial t \partial \tau} \\
\left.\frac{\partial u_{3}}{\partial x}\right|_{x=0}=\left.\frac{\partial u_{3}}{\partial x}\right|_{x=1}=\frac{d}{d t}\left[\left.\gamma u_{0}\right|_{x=1}-\left.u_{0}^{3}\right|_{x=1}\right],
\end{gathered}
$$


решение которой также будем искать в виде (2.9). Непосредственная проверка с учетом (2.16) показывает, что для $C_{n}, n=1,2, \ldots$, здесь получаются аналогичные $(2.10)$ краевые задачи, условия разрешимости которых имеют вид

$$
\frac{i \omega_{n}}{2} \frac{d z_{n}}{d \tau}=-\frac{1}{16} z_{n}+i \omega_{n} f_{n}, \quad n=1,2, \ldots
$$

И наконец, привлекая функцию (2.11), убеждаемся, что, как и раньше, счетная система (2.17) сворачивается в краевую задачу

$$
\frac{1}{2} \frac{\partial^{2} w}{\partial \tau \partial y}=-\frac{1}{16} w+\left[\gamma-3 w^{2}\right] \frac{\partial w}{\partial y}, \quad w(\tau, y+1) \equiv-w(\tau, y) .
$$

При обосновании изложенных формальных построений в случае задачи (1.8) также возникают новые моменты. Для пояснения сути дела введем в рассмотрение характеристическое уравнение

$$
\beta_{0}(1-\operatorname{ch} \mu)+\frac{\mu}{\lambda} \operatorname{sh} \mu=0, \quad \mu=\sqrt{\lambda^{2}+\varepsilon \lambda}
$$

для определения спектра устойчивости ее нулевого состояния равновесия. Несложная проверка показывает, что при условиях (2.14) корни уравнения (2.19) распадаются на две группы: $\lambda_{n}^{1}(\varepsilon), \bar{\lambda}_{n}^{1}(\varepsilon)$ и $\lambda_{n}^{2}(\varepsilon), \bar{\lambda}_{n}^{2}(\varepsilon), n=1,2, \ldots$, причем равномерно по $n$

$$
\lambda_{n}^{1}(\varepsilon)=i \pi(2 n-1)+O\left(\varepsilon^{2}\right), \quad \lambda_{n}^{2}(\varepsilon)=i 2 \pi n-\varepsilon / 2+O\left(\varepsilon^{2}\right) .
$$

Из результатов монографий [24], [25] следует, что первой группе этих корней в задаче (1.8) соответствует бесконечномерное центральное интегральное многообразие, экспоненциально орбитально устойчивое с показателем экспоненты порядка $\varepsilon$. Отметим еше, что указанное многообразие определено, естественно, в некоторой достаточно малой окрестности нулевого положения равновесия, а равенства (2.3), (2.15) (в силу своего построения) задают его асимптотику. Таким образом, система (2.17) представляет собой "укороченную" нормальную форму на данном многообразии, и следовательно, теорема 2.1 здесь остается в силе.

\section{§3. Динамика нормальной формы}

Отдельно остановимся на динамике по параметру $\gamma$ автоколебаний краевых задач (2.13) и (2.18). С этой целью заметим, что обе они при подходящих нормировках переменных $w, \tau, y$ и замене $\tau$ на $t, y$ на $x$ приводятся к виду (2). Таким образом, интересующая нас проблема сводится к аналогичному вопросу для краевой задачи (2). Из теоремы 2.1 следует, что особьй интерес представляют ее периодические решения типа бегуших волн, т.е. периодические решения вида

$$
w=\theta(y), \quad y=\sigma t-x, \quad \sigma>0
$$

Их динамика по $\lambda$ подробно изучена в работах [11], [15]. Поэтому приведем лишш краткий обзор содержащихся там результатов. 
Подставляя (3.1) в задачу (2), для нахождения функции $\theta(y)$ получаем обыкновенное дифференциальное уравнение, которое после замены $y / \sqrt{\sigma} \rightarrow y$ принимает вид

$$
\theta^{\prime \prime}+r\left(\theta^{2}-1\right) \theta^{\prime}+\theta=0
$$

где $r=\lambda / \sqrt{\sigma}$. Заметим, что (3.2) - классическое уравнение Ван-дер-Поля, имеюшее при всех $r>0$ единственное периодическое решение $\theta=H(y, r), H(y, 0)=2 \cos y$, периода $T=T(r), T(0)=2 \pi$, и удовлетворяющее также равенству $H(y+T / 2, r) \equiv$ $-H(y, r)$, Однако в силу того, что фигурирующая в (3.1) функция $\theta(y)$ периодична с периодом 2 , нас интересует решение уравнения (3.2) периода $2 / \sqrt{\sigma}$. Поэтому для определения неизвестного параметра $r$ получаем уравнение

$$
\lambda T(r)-2 r=0 .
$$

При $\lambda \ll 1$, применяя к уравнению (3.3) в точке $\lambda=0, r=0$ теорему о неявной функции, однозначно определяем его решение $r=r_{0}(\lambda), r_{0}(0)=0$. Вспоминая, далее, о связи уравнения (3.2) с исходной задачей (2), заключаем, что последняя имеет периодическое решение

$$
w=\theta_{0}(y, \lambda), \quad y=\sigma_{0}(\lambda) t-x,
$$

где $\sigma_{0}(\lambda)=\left(\lambda / r_{0}(\lambda)\right)^{2}, \theta_{0}(y, \lambda)=H\left(y / \sqrt{\sigma_{0}(\lambda)}, r_{0}(\lambda)\right)$. Отметим также, что, наряду с решением (3.4), у задачи $(2)$ при $\lambda \ll 1$ сушествуют и другие периодические решения, получаюшиеся из построенного с помошью принципа подобия:

$$
w=\theta_{n}(y, \lambda), \quad y=\sigma_{n}(\lambda) t-x, \quad n=0,1,2, \ldots
$$

где $\theta_{n}=\theta_{0}((2 n+1) y,(2 n+1) \lambda), \sigma_{n}(\lambda)=\sigma_{0}((2 n+1) \lambda) /(2 n+1)^{2}$.

Справедливо следующее утверждение (см. [11], [15])

Теорема 3.1. По любому натуральному числу $N$ можсн указать такое достаточно малое $\bar{\lambda}_{N}>0$, что при всех $0<\lambda \leqslant \bar{\lambda}_{N}$ краевая задача (2) имеет экспоненииально орбитально устойчивые периодические решения (3.5) с номерами $n=0,1, \ldots, N$.

Отметим, что найденные решения краевой задачи (2) в форме волн стационарного профиля, описываемых уравнением Ван-дер-Поля, с физической точки зрения близки к автосолитонам, наблюдаемым, например, в кольцевом лазере в случае самосинхронизации мод (см. [26]). При такой интерпретации уравнение (3.3) представляет собой аналог нелинейного дисперсионного соотношения. Отметим еше, что в нашем случае “основной” период колебаний, т.е. период функции $\theta_{0}(y, \lambda)$, равен 2 , а все функции $\theta_{n}(y, \lambda), n \geqslant 1$, в силу принципа подобия имеют периоды $2 /(2 n+1)$. А отсюда и из теоремы 2.1 заключаем, что отвечаюшие решениям (3.5) периодические решения $\left(u_{n}(t, x, \varepsilon), v_{n}(t, x, \varepsilon)\right), n=0,1,2, \ldots$, исходных краевых задач $(1.5),(1.7)$ имеют периоды $T_{n}(\varepsilon)=4 /(2 n+1)+O\left(\varepsilon^{2}\right)$, а аналогичные им периодические решения краевой задачи $(1.8)$ - периоды $T_{n}(\varepsilon)=2 /(2 n+1)+O\left(\varepsilon^{2}\right)$.

Из теоремы 3.1 следует, что при $0<\lambda \ll 1$ в краевой задаче $(2)$ наблюдается явление буферности: существует асимптотически много циклов (3.5), причем можно гарантировать устойчивость любого фиксированного их числа. Далее, как показано 
в [11], [15], каждый цикл (3.5) непрерывно продолжается по $\lambda$ на интервал $0<\lambda<\lambda_{n}$, $\lambda_{n}=\lambda_{0} /(2 n+1), \lambda_{0}=2 /(3-2 \ln 2)$, при $0<\lambda_{n}-\lambda \ll 1$ становится релаксационным (в этом случае стремится к бесконечности параметр $r$ в (3.2)), а при $\lambda \geqslant \lambda_{n}-$ разрывным. С физической точки зрения это вполне понятно: увеличение энергетического параметра $\lambda$ в совокупности с эффектом самосинхронизации мод приводит сначала к усложнению формы каж дого цикла (3.5), а затем (при $\left.\lambda \geqslant \lambda_{n}\right)-$ к полному его разрушению.

Способ исчезновения цикла (3.5) при прохождении $\lambda$ через значение $\lambda_{n}$ уместно назвать градиентной катастрофой в силу того, что при $\lambda \rightarrow \lambda_{n}-0$ стремится к бесконечности его $C^{1}$-норма, в то время как $C$-норма остается ограниченной. Данный феномен для периодических решений типа бегуших волн у нелинейного телеграфного уравнения отмечался ранее в работе [27]. Там, в частности, показано, что в момент градиентной катастрофы цикл может быть устойчивым.

Вопрос об аттракторах краевой задачи (2) при $\lambda>\lambda_{0}$ остается открытым. Наиболее вероятным здесь представляется следуюшее: все ее решения $w(t, x) \not \equiv 0$ при $t \rightarrow \infty$, оставаясь ограниченными в $C$-норме, стремятся к разрывным функциям.

Подведем некоторые итоги. Заметим. во-первых, что спектр собственных частот краевых задач (1.5), (1.7), (1.8) при условиях (2.1), (2.14) и при $\varepsilon=0$ является, очевидно, эквидистантным. Во-вторых, как следует из теорем 2.1, 3.1 и из описанной выше динамики нормальной формы (2) по $\lambda$, при увеличении энергетического параметра $\gamma$ в каждой из этих задач реализуется схема (1), т.е. происходит переход от буферности к разрывным колебаниям. Таким образом, получила подтверждение основная из отмеченных во Введении альтернатив, при которых имеем дело с модельной краевой задачей (2). Отметим еше, что диапазон изменения коэффищиента усиления $\beta_{0}$, в котором наблюдается явление буферности, в этих задачах весьма узок - имеет порядок $\varepsilon^{2}$.

\section{§. Метод квазинормальных форм}

С физической точки зрения переход в краевых задачах (1.5), (1.7), (1.8) от гладких колебаний к разрывным при увеличении коэффициента усиления означает, что в этом случае мы выходим за рамки применимости данных математических моделей. Поэтому естественно возникает вопрос о сглаживании разрьвных колебаний путем учета каких-то дополнительных факторов. Как известно [22], подобная проблема решается введением паразитных индуктивностей, емкостей и т.д. Здесь мы проиллюстрируем указанньй подход на примере автогенератора, описьваемого задачей (1.7).

Рассмотрим краевую задачу

$$
\begin{array}{ll}
u_{t}=-v_{x}, & v_{t}=-u_{x}-\varepsilon v-\left.\varepsilon\left[\beta_{0} u+\beta_{1} u^{2}-\beta_{2} u^{3}\right]\right|_{x=1}, \\
\left.u\right|_{x=0}=0, & \left.\alpha v_{x}\right|_{x=1}+\left.v\right|_{x=1}+\left.\beta_{0} u\right|_{x=1}+\left.\beta_{1} u^{2}\right|_{x=1}-\left.\beta_{2} u^{3}\right|_{x=1}=0
\end{array}
$$

получающуюся из (1.7) при учете входной паразитной емкости $C_{0}$ нелинейного элемента $\left(\alpha=C_{0} /(l C)\right)$. В качестве ее фазового пространства возьмем $(u, v) \in \stackrel{\circ}{W} \stackrel{1}{2}(0,1) \times$ $W_{2}^{1}(0,1)$, где $\stackrel{\circ}{W} \underset{2}{1} \subset W_{2}^{1}$ - подпространство функций, удовлетворяющих первому граничному условию из (4.1). Отличие от исходной задачи (1.7) заключается в том, что 
за счет слагаемого $\left.\alpha v_{x}\right|_{x=1}$ отпадает необходимость в требовании вьполнения для начальных функций второго граничного условия (4.1).

Автоколебаниями задачи (4.1) будем интересоваться при $0<\varepsilon \ll 1,0<\alpha \ll 1$ и при условиях

$$
\beta_{0}=\varepsilon \beta, \quad \beta_{1}=0, \quad \beta_{2}=1,
$$

где параметр $\beta>0$ имеет порядок единицы. Далее, для согласования порядков малости $\varepsilon$ и $\alpha$ заметим, что, во-первых, при условиях (4.2) и при $\varepsilon=0$ спектр устойчивости нулевого положения равновесия задачи (4.1) состоит из чисто мнимых собственных значений $\pm i \sigma_{n}$, где $\sigma_{1}<\sigma_{2}<\cdots<\sigma_{n}<\cdots-$ положительные корни уравнения $\operatorname{ctg} \sigma=\alpha \sigma$; во-вторых, при каждом фиксированном $n$ справедливо асимптотическое представление

$$
\sigma_{n}=\omega_{n}\left(1-\alpha+\alpha^{2}\right)+\left(\omega_{n}^{3} / 3-\omega_{n}\right) \alpha^{3}+O\left(\alpha^{4}\right),
$$

где $\omega_{n}=\pi(2 n-1) / 2, n=1,2, \ldots$. Формулы (4.3) наводят на мысль о целесообразности условия

$$
\alpha=\alpha_{0} \varepsilon^{1 / 3}, \quad \alpha_{0}=\text { const }>0,
$$

так как в этом случае “отход” от резонансности у собственных частот $\sigma_{n}$ происходит в порядке $\varepsilon$, что согласуется с порядком малости коэффициента усиления $\beta_{0}$ в $(4.2)$.

Итак, исследованию подлежит краевая задача

$$
\begin{aligned}
& u_{t}=-v_{x}, \quad v_{t}=-u_{x}-\varepsilon v \\
& \left.u\right|_{x=0}=0,\left.\quad \varepsilon^{1 / 3} \alpha_{0} v_{x}\right|_{x=1}+\left.v\right|_{x=1}+\left.\varepsilon\left[\beta u-u^{3}\right]\right|_{x=1}=0,
\end{aligned}
$$

получающаяся из (4.1) при условиях (4.2), (4.4) после нормировок $u / \sqrt{\varepsilon} \rightarrow u$, $v / \sqrt{\varepsilon} \rightarrow v$ и отбрасывания слагаемых порядка малости $\varepsilon^{2}$. Для построения ее автоколебаний снова воспользуемся формализмом метода бесконечномерной нормализации, которьй в данном случае имеет определенную специфику. А именно, вместо (2.3) подставим в задачу (4.5) ряды

$$
\begin{aligned}
& u=u_{0}(\tau, s, x)+\varepsilon^{1 / 3} u_{1}(\tau, s, x)+\varepsilon^{2 / 3} u_{2}(\tau, s, x)+\varepsilon u_{3}(\tau, s, x)+\cdots \\
& v=v_{0}(\tau, s, x)+\varepsilon^{1 / 3} v_{1}(\tau, s, x)+\varepsilon^{2 / 3} v_{2}(\tau, s, x)+\varepsilon v_{3}(\tau, s, x)+\cdots
\end{aligned}
$$

где

$$
\begin{gathered}
\tau=\left(1-\alpha_{0} \varepsilon^{1 / 3}+\alpha_{0}^{2} \varepsilon^{2 / 3}\right) t, \quad s=\varepsilon t \\
u_{0}=\sum_{n=1}^{\infty}\left[z_{n}(s) \exp \left(i \omega_{n} \tau\right)+\bar{z}_{n}(s) \exp \left(-i \omega_{n} \tau\right)\right](-1)^{n-1} \sin \omega_{n} x \\
v_{0}=i \sum_{n=1}^{\infty}\left[z_{n}(s) \exp \left(i \omega_{n} \tau\right)-\bar{z}_{n}(s) \exp \left(-i \omega_{n} \tau\right)\right](-1)^{n-1} \cos \omega_{n} x \\
u_{j}=\sum_{n=1}^{\infty}\left(A_{n}^{j}(s, x) \exp \left(i \omega_{n} \tau\right)+\bar{A}_{n}^{j}(s, x) \exp \left(-i \omega_{n} \tau\right)\right) \\
v_{j}=\sum_{n=1}^{\infty}\left(B_{n}^{j}(s, x) \exp \left(i \omega_{n} \tau\right)+\bar{B}_{n}^{j}(s, x) \exp \left(-i \omega_{n} \tau\right)\right)
\end{gathered}
$$


Здесь $j=1,2,3, \omega_{n}=\pi(2 n-1) / 2$, а неизвестные комплексные амплитуды $z_{n}$ таковы, что по-прежнему сходится ряд с обшим членом $\omega_{n}^{2}\left|z_{n}\right|^{2}$.

Приравнивая в (4.5) коэффишиенты при $\varepsilon^{1 / 3}, \varepsilon^{2 / 3}$, для определения фигурируюших в (4.9) коэффициентов $A_{n}^{j}, B_{n}^{j}, j=1,2$, получаем краевые задачи

$$
\begin{array}{ll}
\frac{d B_{n}^{1}}{d x}+i \omega_{n} A_{n}^{1}=\alpha_{0} i \omega_{n} z_{n}(-1)^{n-1} \sin \omega_{n} x, & \left.A_{n}^{1}\right|_{x=0}=0, \\
\frac{d A_{n}^{1}}{d x}+i \omega_{n} B_{n}^{1}=-\alpha_{0} \omega_{n} z_{n}(-1)^{n-1} \cos \omega_{n} x, & \left.B_{n}^{1}\right|_{x=1}=i z_{n} \alpha_{0} \omega_{n} ; \\
\frac{d B_{n}^{2}}{d x}+i \omega_{n} A_{n}^{2}=\alpha_{0} i \omega_{n} A_{n}^{1}-\alpha_{0}^{2} i \omega_{n} z_{n}(-1)^{n-1} \sin \omega_{n} x, & \left.A_{n}^{2}\right|_{x=0}=0, \\
\frac{d A_{n}^{2}}{d x}+i \omega_{n} B_{n}^{2}=\alpha_{0} i \omega_{n} B_{n}^{1}+\alpha_{0}^{2} \omega_{n} z_{n}(-1)^{n-1} \cos \omega_{n} x, & \left.B_{n}^{2}\right|_{x=1}=-\left.\alpha_{0} \frac{d B_{n}^{1}}{d x}\right|_{x=1},
\end{array}
$$

которые оказываются разрешимыми за счет специального выбора поправок порядка $\varepsilon^{1 / 3}$ и $\varepsilon^{2 / 3}$ в (4.7). Несложньй их анализ приводит к равенствам

$$
\begin{gathered}
A_{n}^{1}=-\alpha_{0}(-1)^{n-1} \omega_{n} z_{n} x \cos \omega_{n} x, \quad B_{n}^{1}=i \alpha_{0}(-1)^{n-1} \omega_{n} z_{n} x \sin \omega_{n} x \\
A_{n}^{2}=\alpha_{0}^{2}(-1)^{n-1} \omega_{n} z_{n}\left(x \cos \omega_{n} x-\frac{\omega_{n} x^{2}}{2} \sin \omega_{n} x\right) \\
B_{n}^{2}=-\alpha_{0}^{2}(-1)^{n-1} i \omega_{n} z_{n}\left(x \sin \omega_{n} x+\frac{\omega_{n} x^{2}}{2} \cos \omega_{n} x\right) .
\end{gathered}
$$

На третьем шаге после исключения $A_{n}^{3}$ приходим к краевым задачам

$$
\begin{gathered}
\frac{d^{2} B_{n}^{3}}{d x^{2}}+\omega_{n}^{2} B_{n}^{3}=2 \alpha_{0} \omega_{n}^{2} B_{n}^{2}-3 \alpha_{0}^{2} \omega_{n}^{2} B_{n}^{1}+2 \alpha_{0}^{3} i \omega_{n}^{2} z_{n}(-1)^{n-1} \cos \omega_{n} x \\
-\omega_{n}(-1)^{n-1} z_{n} \cos \omega_{n} x-2 \omega_{n} \frac{d z_{n}}{d s}(-1)^{n-1} \cos \omega_{n} x \\
\left.\frac{d B_{n}^{3}}{d x}\right|_{x=0}=0,\left.\quad B_{n}^{3}\right|_{x=1}=-\left.\alpha_{0} \frac{d B_{n}^{2}}{d x}\right|_{x=1}-f_{n} .
\end{gathered}
$$

Здесь $f_{n}-$ коэффициент при $\exp \left(i \omega_{n} y\right)$ ряда Фурье функции $\beta w-w^{3}$, где

$$
w(s, y)=\sum_{n=1}^{\infty}\left(z_{n}(s) \exp \left(i \omega_{n} y\right)+\bar{z}_{n}(s) \exp \left(-i \omega_{n} y\right)\right)
$$

Условия разрешимости задач (4.12) с учетом (4.10), (4.11) приводят, в свою очередь, к счетной системе уравнений

$$
\frac{d z_{n}}{d s}=-z_{n} / 2+i \alpha_{0}^{3}\left[\omega_{n}^{3} / 3-\omega_{n}\right] z_{n}+f_{n}, \quad n=1,2, \ldots,
$$

для нахождения амплитуд $z_{n}$. И наконец, привлекая функцию (4.13), убеждаемся, что система (4.14) сворачивается в краевую задачу

$$
\frac{\partial w}{\partial s}=-\frac{\alpha_{0}^{3}}{3} \frac{\partial^{3} w}{\partial y^{3}}-\alpha_{0}^{3} \frac{\partial w}{\partial y}+(\beta-1 / 2) w-w^{3}, \quad w(s, y+2) \equiv-w(s, y) .
$$


Подчеркнем, что задача (4.15) не является нормальной формой для исходной задачи (4.5), ибо не сушествует корректно определенной замены, приводящей последнюю к виду (4.14). Для пояснения сути дела рассмотрим пространство $l_{2}^{1}$ последовательностей вида $z=\left(z_{1}, \bar{z}_{1}, \ldots, z_{n}, \bar{z}_{n}, \ldots\right)$, для которых конечна норма $\|z\|=$ $\left(\sum_{n=1}^{\infty} \omega_{n}^{2}\left|z_{n}\right|^{2}\right)^{1 / 2}$, и заметим, что построенные в $\S 2$ отрезки рядов (2.3) индуцируют обратимьй ограниченный оператор, действующий из $l_{2}^{1}$ в $W_{2}^{1}\left([0,1] ; \mathbb{R}^{2}\right)$. А это, в свою очередь, позволяет сконструировать на них корректную замену переменных, приводящую задачу (2.2) к системе в пространстве $l_{2}^{1}$, имеющей с точностью до слагаемых порядка $\varepsilon$ вид (2.12). В случае же построенных вьше отрезков рядов (4.6) ситуация иная: при произвольно фиксированном векторе $z \in l_{2}^{1}$, как следует из (4.10), имеем $u_{1}, v_{1} \in L_{2}(0,1)$ по переменной $x$, а ряды для $u_{2}, v_{2}$ и тем более для $u_{3}, v_{3}$ вообше носят формальный характер. Поэтому согласно введенной в [17], [18] терминологии краевую задачу (4.15) будем называть квазинормальной формой.

Несмотря на все вьшесказанное, стандартное утверждение о соответствии между периодическими решениями задач (4.15) и (4.5) остается в силе. Точнее говоря, каждому периодическому решению $w=w_{0}(\xi), \xi=\sigma_{0} s+y, \sigma_{0}=$ const, задачи (4.15), экспоненциально орбитально устойчивому или дихотомичному, при всех достаточно малых $\varepsilon>0$ в исходной задаче (4.5) соответствует цикл той же устойчивости, а его асимптотика получается из (4.6)-(4.9) по описанному в теореме 2.1 правилу. Доказательство этого утверждения вытекает из результатов работ [18] и [11], [28], где даны две различные версии обоснования метода квазинормальных форм в гиперболическом случае.

Отметим, что при $\beta<1 / 2$ нулевое решение устойчиво как в исходной задаче (4.5), так и в рамках ее квазинормальной формы (4.15). Поэтому ниже динамикой последней будем интересоваться при $\beta>1 / 2$. В этом случае после замены $y-\alpha_{0}^{3} s \rightarrow y$, убивающей слагаемое $-\alpha_{0}^{3} \partial w / \partial y$, подходяших нормировок переменных $s, y, w$ и переобозначений $s \rightarrow t, y \rightarrow x$ задача (4.15) приводит к виду (3), где $a^{2}=\alpha_{0}^{3} /[24(\beta-1 / 2)]$.

Остановимся на вопросе о существовании и устойчивости у краевой задачи (3) периодических решений

$$
w=w(y), \quad y=(2 n+1) x+a^{2}(2 n+1)^{3} \sigma t, \quad n=0,1, \ldots .
$$

Подставляя (4.16) в (3), для определения антипериодической с периодом 1 функции $w(y)$ и постоянной $\sigma$ приходим к дифференциальному уравнению

$$
w^{\prime \prime \prime}+\sigma w^{\prime}=\mu\left[w-w^{3}\right]
$$

в котором параметр $\mu=a^{-2}(2 n+1)^{-3}$ для удобства будем считать меняющимся непрерьвно. Справедливо следующее утверждение [10], [11].

TEOPEмA 4.1. Найдется такое достаточно малое $\mu_{0}>0$, что при всех $0<\mu \leqslant \mu_{0}$ существует единственная пара достаточно гладких по своим переменным функиий

$$
\begin{gathered}
(\sigma(\mu), w(y, \mu)): w(y+1, \mu) \equiv-w(y, \mu), \quad \sigma(0)=\pi^{2}, \quad \sigma^{\prime}(0)=0, \\
w(y, 0)=\frac{2}{\sqrt{3}} \cos \pi y, \quad w(1 / 2, \mu) \equiv 0,
\end{gathered}
$$


обращающих уравнение (4.17) в тождество.

Сформулированная теорема доставляет сразу счетное число периодических решений

$$
w=w(y, \mu), \quad y=(2 n+1) x+a^{2}(2 n+1)^{3} \sigma(\mu) t, \quad \mu=\left[a^{2}(2 n+1)^{3}\right]^{-1},
$$

задачи (3) с номерами $n$, удовлетворяющими неравенству

$$
\left[a^{2}(2 n+1)^{3}\right]^{-1} \leqslant \mu_{0}
$$

Их устойчивость также исследована в работах [10], [11], где получено следуюшее утверждение.

ТЕОРема 4.2. Все периодические решения (4.18) экспоненииально орбитально устойчивъ (в метрике пространства $W_{2}^{3}$ антипериодических с периодом 1 функиий $w(y))$.

Теоремы 4.1 и 4.2 описьвают явление гипербуферности (см. Введение), реализующееся в краевой задаче (3) при любом значении параметра $a$. Отметим еше, что при $a \gg 1$ неравенство (4.19) заведомо вьполняется для всех номеров $n=0,1, \ldots$, а значит, в этом случае имеется полньй набор циклов (4.16). Можно показать также, что при $a \gg 1$ имеются и торы всевозможных размерностей. Все они, однако, неустойчивы.

В случае $a \sim 1$ теоремы 4.1 и 4.2 гарантируют существование и устойчивость только циклов (4.18) с номерами, большими некоторого $n_{0}$. Циклы, отвечаюшие меньшим номерам $n$, становятся нелокальными, т.е. усложняется их зависимость от $t, x$. И наконец, обрашаясь к исходной задаче (4.5) и опираясь на аналог теоремы 2.1, заключаем, что при всех $0<\varepsilon \ll 1$ в ней также наблюдается феномен буферности, однако речь здесь должна идти лишь о произвольном конечном числе устойчивых циклов.

Итак, краевая задача (4.5) представляет собой требуемую регуляризацию математической модели (1.7), о необходимости которой говорилось в начале этого параграфа. Действительно, при $\alpha=0$ и при условиях (4.2) краевая задача (4.1) не может адекватно описывать работу реального физического объекта, так как при $\beta>1 / 2$ все нетривиальные решения ее квазинормальной формы (4.15) (в которой теперь следует положить $\alpha_{0}=0$ ) стремятся при $s \rightarrow \infty$ к разрывным функциям. С учетом же малой паразитной емкости происходит регуляризация разрывных колебаний, причем сохраняется явление буферности.

\section{$\S$ 5. Случай неэквидистантного спектра}

В этом параграфе проведем исследование автоколебаний краевой задачи (4.1) при условии (4.2) и при $\alpha \sim 1$. В данном случае спектр ее собственных частот $\omega_{n}, n \geqslant 1$, неэквидистантен, так как $\omega_{n}$ - это занумерованные в порядке возрастания положительные корни уравнения $\operatorname{ctg} \omega=\alpha \omega$. В подобной ситуации, как известно (см. [11], $[12],[16])$, периодические решения имеют квазигармоническую форму, а их асимптотика строится с помощью классического одночастотного метода [23], применяемого к каждой частоте в отдельности. 
Итак, анализу подлежит аналогичная (4.5) краевая задача

$$
\begin{array}{ll}
u_{t}=-v_{x}, & v_{t}=-u_{x}-\varepsilon v, \\
\left.u\right|_{x=0}=0, & \left.\alpha v_{x}\right|_{x=1}+\left.v\right|_{x=1}+\left.\varepsilon\left[\beta u-u^{3}\right]\right|_{x=1}=0,
\end{array}
$$

где $0<\varepsilon \ll 1$, а параметры $\alpha, \beta>0$ имеют порядок единицы. Следуя методике работы [12], фиксируем произвольно номер $n$ и подставим в (5.1) ряды

$$
\begin{gathered}
u=u_{0}(\tau, x)+\varepsilon u_{1}(\tau, x)+\cdots, \quad v=v_{0}(\tau, x)+\varepsilon v_{1}(\tau, x)+\cdots \\
\tau=\left(1+\varepsilon \delta_{1}+\varepsilon^{2} \delta_{2}+\cdots\right) t
\end{gathered}
$$

Здесь $u_{j}(\tau, x), v_{j}(\tau, x), j \geqslant 0,-$ подлежашие определению периодические по $\tau$ с периодом $2 \pi / \omega_{n}$ функции, причем

$$
\begin{aligned}
& u_{0}=\xi\left[\exp \left(i \omega_{n} \tau\right)+\exp \left(-i \omega_{n} \tau\right)\right] \sin \omega_{n} x \\
& v_{0}=i \xi\left[\exp \left(i \omega_{n} \tau\right)-\exp \left(-i \omega_{n} \tau\right)\right] \cos \omega_{n} x
\end{aligned}
$$

а $\xi, \delta_{j}, j \geqslant 1,-$ произвольные вешественные параметры, также подлежашие определению. Подчеркнем, что отыскание интересуюших нас периодических решений в виде (5.2) вполне естественно, так как равенствами (5.3) с заменой $\tau$ на $t$ задается произвольное $2 \pi / \omega_{n}$-периодическое решение задачи (5.1) при $\varepsilon=0$.

Приравнивая в (5.1) коэффициенты при $\varepsilon$, для определения $u_{1}, v_{1}$ получаем линейную неоднородную краевую задачу, которая после исключения $u_{1}$ принимает вид

$$
\begin{gathered}
\left(\frac{\partial^{2}}{\partial \tau^{2}}-\frac{\partial^{2}}{\partial x^{2}}\right) v_{1}=-2 \delta_{1} \frac{\partial^{2} v_{0}}{\partial \tau^{2}}-\frac{\partial v_{0}}{\partial \tau} \\
\left.\frac{\partial v_{1}}{\partial x}\right|_{x=0}=0,\left.\quad \alpha \frac{\partial v_{1}}{\partial x}\right|_{x=1}+\left.v_{1}\right|_{x=1}=\left.\left[u_{0}^{3}-\beta u_{0}\right]\right|_{x=1} .
\end{gathered}
$$

Ее решение будем искать в том же виде, что и соответствующие неоднородности, т.е. в виде тригонометрического многочлена

(5.5) $v_{1}=A(x) \exp \left(i \omega_{n} \tau\right)+\bar{A}(x) \exp \left(-i \omega_{n} \tau\right)+B(x) \exp \left(3 i \omega_{n} \tau\right)+\bar{B}(x) \exp \left(-3 i \omega_{n} \tau\right)$.

Подставляя (5.5) в (5.4), для нахождения $A, B$ приходим к краевым задачам

$$
\begin{gathered}
A^{\prime \prime}+\omega_{n}^{2} A=-\xi \omega_{n}\left(1+2 i \delta_{1} \omega_{n}\right) \cos \omega_{n} x, \\
A^{\prime}(0)=0, \quad \alpha A^{\prime}(1)+A(1)=3 \xi^{3} \sin ^{3} \omega_{n}-\beta \xi \sin \omega_{n} ; \\
B^{\prime \prime}+9 \omega_{n}^{2} B=0, \\
B^{\prime}(0)=0, \quad \alpha B^{\prime}(1)+B(1)=\xi^{3} \sin ^{3} \omega_{n} .
\end{gathered}
$$

Краевая задача (5.6) является вырожденной, а условие ее разрешимости задается равенством

$$
-\frac{\xi}{2}\left(1+2 i \delta_{1} \omega_{n}\right)\left(1+\alpha+\alpha^{2} \omega_{n}^{2}\right)+\beta \xi-3 \xi^{3} \sin ^{2} \omega_{n}=0
$$


Предположим, далее, что выполняется неравенство

$$
\beta>\beta_{n},
$$

где $\beta_{n}=\left(1+\alpha+\alpha^{2} \omega_{n}^{2}\right) / 2$. Тогда из (5.8) находим

$$
\delta_{1}=0, \quad \xi=\sqrt{\left(\beta-\beta_{n}\right) /\left(3 \sin ^{2} \omega_{n}\right)},
$$

а затем, решая задачи (5.6) и (5.7) (вторая из них - невырожденная), окончательно получаем

$$
A(x)=-\frac{\xi}{2} x \sin \omega_{n} x, \quad B(x)=-\frac{\xi^{3}}{8 \alpha^{3} \omega_{n}^{3}} \cos 3 \omega_{n} x .
$$

И наконец, компонента $u_{1}$ определяется в виде тригонометрического многочлена структуры (5.5) из уравнения $\partial u_{1} / \partial \tau=-\partial v_{1} / \partial x$.

Как следует из [11], в принципе полученной информации достаточно для доказательства сушествования у задачи (5.1) периодического решения с нулевым приближением, задающимся равенствами (5.3), (5.10). Однако при необходимости описанньй алгоритм можно продолжить. При этом разрешимости соответствующих линейных неоднородных краевых задач для $u_{j}(\tau, x), v_{j}(\tau, x), j \geqslant 1$, в классе нечетных тригонометрических полиномов аргумента $\omega_{n} \tau$ добиваемся за счет слагаемых вида (5.3), с точностью до которых определяются функции $u_{j-1}, v_{j-1}$, и очередной поправки к частоте $\delta_{j}$.

Следующий этап - исследование свойств устойчивости построенного выше приближенного периодического решения. Линеаризуя на нем задачу (5.1) и отбрасывая слагаемые порядка малости $\varepsilon^{2}$ и выше, приходим к линейной краевой задаче

$$
\begin{gathered}
u_{t}=-v_{x}, \quad v_{t}=-u_{x}-\varepsilon v \\
\left.u\right|_{x=0}=0,\left.\quad \alpha v_{x}\right|_{x=1}+\left.v\right|_{x=1}+\left.\varepsilon a(t) u\right|_{x=1}=0
\end{gathered}
$$

где

$$
a(t)=\beta-3 \xi^{2}\left[2+\exp \left(2 i \omega_{n} t\right)+\exp \left(-2 i \omega_{n} t\right)\right] \sin ^{2} \omega_{n},
$$

а $\xi$ задается в (5.10).

Следуя в очередной раз методике работы [12], при $m=1,2, \ldots, m \neq n$, подставим в (5.11)

$$
\begin{aligned}
& u=\left[\sin \omega_{m} x+\varepsilon z_{m}(t, x)\right] \exp \left(i \omega_{m}+\varepsilon \lambda_{m}\right) t \\
& v=\left[i \cos \omega_{m} x+\cos \omega_{m} x+\varepsilon w_{m}(t, x)\right] \exp \left(i \omega_{m}+\varepsilon \lambda_{m}\right) t
\end{aligned}
$$

где постоянная $\lambda_{m}$ и функции $z_{m}, w_{m}$, периодические по $t$ с периодом $2 \pi / \omega_{n}$, подлежат определению. Приравнивая затем коэффициенты при $\varepsilon$, для $z_{m}, w_{m}$ получаем линейную неоднородную краевую задачу, которая после исключения $z_{m}$ приобретает вид

$$
\begin{gathered}
\frac{\partial^{2} w_{m}}{\partial t^{2}}+2 i \omega_{m} \frac{\partial w_{m}}{\partial t}-\omega_{m}^{2} w_{m}-\frac{\partial^{2} w_{m}}{\partial x^{2}}=\omega_{m}\left(2 \lambda_{m}+1\right) \cos \omega_{m} x, \\
\left.\frac{\partial w_{m}}{\partial x}\right|_{x=0}=0,\left.\quad \alpha \frac{\partial w_{m}}{\partial x}\right|_{x=1}+\left.w_{m}\right|_{x=1}+a(t) \sin \omega_{m}=0 .
\end{gathered}
$$


Из структуры фигурируюшей в задаче (5.14) неоднородности (см. (5.12)) заключаем, что ее решение следует искать в форме

$$
w_{m}=C_{0, m}(x)+C_{1, m}^{+}(x) \exp \left(2 i \omega_{n} t\right)+C_{1, m}^{-}(x) \exp \left(-2 i \omega_{n} t\right) .
$$

Подставляя, далее, $(5.15)$ в (5.14), для $C_{0, m}$ получаем аналогичную $(5.6)$ вырожденную краевую задачу, разрешимости которой добиваемся выбором $\lambda_{m}$. Соответствующий анализ приводит к равенствам

$$
C_{0, m}=-\frac{1}{2}\left(2 \lambda_{m}+1\right) x \sin \omega_{m} x, \quad \lambda_{m}=\left[2 \beta_{n}-\beta_{m}-\beta\right] /\left(2 \beta_{m}\right) .
$$

В случае же $C_{1, m}^{ \pm}$имеем дело с невырожденными краевыми задачами, из которых определяем:

$$
C_{1, m}^{ \pm}(x)=\mp \frac{3 \xi^{2}}{2 \alpha^{3} \omega_{n}\left(\omega_{m} \pm \omega_{n}\right)^{2}} \cos \left(\omega_{m} \pm 2 \omega_{n}\right) x .
$$

При $m=n$, т.е. на собственной частоте $\omega_{n}$ периодического решения, описанньй выше алгоритм приходится несколько видоизменить. Связано это с тем, что при $m=n$ обрашается в нуль знаменатель в формуле для $C_{1, m}^{-}($см $(5.17))$. Поэтому здесь, опираясь на методику работы [29], вместо (5.13) подставим в (5.11)

$$
u=\left[Z_{0}(t, x)+\varepsilon Z_{1}(t, x)\right] \exp (\varepsilon D t), \quad v=\left[W_{0}(t, x)+\varepsilon W_{1}(t, x)\right] \exp (\varepsilon D t),
$$

где

$$
\begin{aligned}
Z_{0}(t, x) & =\left[\exp \left(i \omega_{n} t\right) \sin \omega_{n} x, \exp \left(-i \omega_{n} t\right) \sin \omega_{n} x\right] \\
W_{0}(t, x) & =\left[\exp \left(i \omega_{n} t\right) i \cos \omega_{n} x,-\exp \left(-i \omega_{n} t\right) i \cos \omega_{n} x\right]
\end{aligned}
$$

а элементы $2 \pi / \omega_{n}$-периодических по $t$ матрищ-строк

$$
Z_{1}(t, x)=\left[z_{1, n}, \bar{z}_{1, n}\right], \quad W_{1}(t, x)=\left[w_{1, n}, \bar{w}_{1, n}\right]
$$

и постоянной матрицы $D=\left(d_{j k}\right), j, k=1,2$, подлежат определению. Действуя, далее, указанным выше образом, для функций $z_{1, n}, w_{1, n}$ получим линейную неоднородную краевую задачу, из условий разрешимости которой в классе тригонометрических полиномов аргумента $\omega_{n} t$ находим элементы матрицы $D$. Опуская вполне понятные вькладки, приведем сразу окончательньй результат:

$$
D=\left(\begin{array}{ll}
d & d \\
d & d
\end{array}\right), \quad d=\frac{\beta_{n}-\beta}{2 \beta_{n}}
$$

Отметим, что одно собственное значение матрищы (5.18) - нулевое (ибо (5.11) - это линеаризация на цикле), а второе в силу (5.9) отрицательно.

Обоснование описанных алгоритмов и доказательство существования у краевой задачи (5.1) периодического решения с асимптотикой (5.2) проводятся по стандартньм схемам (см. [11]). В нашем случае из проделанных вьше построений и из результатов [11] вытекает следуюшее утверждение. 
ТЕОРема 5.1. Пусть выполнено неравенство (5.9), отличны от нуля все числа $\lambda_{m}$ из (5.16) и количество положстельных среди них равно $m_{0}$. Тогда найдется такое достаточно малое $\varepsilon_{0}>0$, что при всех $0<\varepsilon \leqslant \varepsilon_{0}$ краевая задача (5.1) имеет иикл (периодическое по $t$ решение) с нулевым приближением (5.3), (5.10). Данный цикл әкспоненциально орбитально устойчив при $m_{0}=0$ и дихотомичен при $m_{0}>0$ с размерностью неустойчивого многообразия, равной $2 m_{0}+1$.

Заметим, что число $m_{0}$ всегда конечно, поскольку $\lambda_{m} \rightarrow-1 / 2$ при $m \rightarrow \infty$. Отметим, далее, что, как следует из приведенной теоремы, цикл (5.2) с номером $n=1$ устойчив при всех $\beta>\beta_{1}$. Циклы же с номерами $n \geqslant 2$, бифурцируюшие из нуля при $\beta=\beta_{n}$, сначала, т.е. при $\beta_{n}<\beta<2 \beta_{n}-\beta_{1}$, неустойчивы, но становятся устойчивыми при дальнейшем увеличении параметра $\beta$, а именно, при

$$
\beta>2 \beta_{n}-\beta_{1}
$$

Тем самым, при подходяшем увеличении $\beta$ можно гарантировать сушествование у краевой задачи (5.1) любого наперед заданного числа устойчивых циклов, т.е. наблюдается феномен буферности.

Итак, краевая задача (5.1) иллюстрирует описанную во Введении типовую ситуацию, когда в гиперболической системе с неэквидистантным спектром собственных частот при увеличении энергетических параметров реализуется явление буферности. Далее, при $\alpha \rightarrow 0$, т.е. при переходе в (5.1) от неэквидистантного спектра к эквидистантному, и при любом фиксированном $\beta>1 / 2$ условия самовозбуждения (5.9) и устойчивости (5.19) начинают выполняться для все большего количества частот. Наконец, при $\alpha \sim \varepsilon^{1 / 3}$ часть устойчивых циклов (на младших модах) теряет гармоническую форму: в этом случае процесс описьвается модельной краевой задачей (3). Таким образом, получил подтверждение и отмеченньй во Введении сценарий перехода от неэквидистантного спектра к эквидистантному, связанный с сохранением феномена буферности.

\section{§. Гибридная краевая задача}

Из приведенных вьше результатов следует, что базовые краевые задачи (2) и (3) при определенных условиях могут описьвать автоколебания в рамках одного и того же автогенератора. Поэтому естественно возникает вопрос о нахождении такой универсальной математической модели, анализ которой приводил бы к некоторой гибридной краевой задаче, включающей задачи (2) и (3) как частные случаи. Как будет показано в этом параграфе, в качестве такой математической модели можно взять краевую задачу

$$
\begin{array}{ll}
u_{t}=-v_{x}, & v_{t}=-u_{x}-\varepsilon v-\left.\varepsilon\left[\beta_{0} u-u^{3}\right]\right|_{x=1}, \\
\left.u\right|_{x=0}=0,\left.\quad \varepsilon^{2 / 3} \alpha_{0} v_{x}\right|_{x=1}+\left.v\right|_{x=1}+\left.\left[\beta_{0} u-u^{3}\right]\right|_{x=1}=0
\end{array}
$$

получающуюся из (1.7) при учете малой паразитной емкости $C_{0} /(l C)=\varepsilon^{2 / 3} \alpha_{0}, \alpha_{0}=$ const $>0$, и специальном выборе коэффициента усиления

$$
\beta_{0}=\varepsilon / 2+\alpha_{0} \varepsilon^{5 / 3} / 2+\varepsilon^{2} \gamma, \quad \gamma=\text { const }>0 .
$$


Для построения автоколебаний задачи (6.1) при условии (6.2) воспользуемся в очередной раз формализмом метода бесконечномерной нормализации. В данном случае подставим в (6.1) ряды

$$
\begin{aligned}
& u=\varepsilon u_{0}+\varepsilon^{5 / 3} u_{1}+\varepsilon^{2} u_{2}+\varepsilon^{7 / 3} u_{3}+\varepsilon^{8 / 3} u_{4}+\varepsilon^{3} u_{5}+\cdots, \\
& v=\varepsilon v_{0}+\varepsilon^{5 / 3} v_{1}+\varepsilon^{2} v_{2}+\varepsilon^{7 / 3} v_{3}+\varepsilon^{8 / 3} v_{4}+\varepsilon^{3} v_{5}+\cdots,
\end{aligned}
$$

где

$$
\begin{aligned}
u_{j}=u_{j}(\tau, s, x), \quad v_{j}=v_{j}(\tau, s, x), & j=0,1, \ldots, 5, \\
\tau=\left(1-\alpha_{0} \varepsilon^{2 / 3}+\alpha_{0}^{2} \varepsilon^{4 / 3}\right) t, & s=\varepsilon^{2} t
\end{aligned}
$$

а функции $u_{0}, v_{0}$ заданы в (4.8). Действуя, далее, обычньм образом, для $u_{1}, v_{1}$ приходим к формулам (4.9), (4.10), для $u_{2}, v_{2}-$ к формулам (2.6), (2.7), где $t$ следует заменить на $\tau$, а для $u_{3}, v_{3}-$ к формулам (4.9) (при $\left.j=2\right),(4.11)$.

Некоторый новый момент возникает на четвертом шаге. Здесь после исключения $u_{4}$ имеем дело с краевой задачей

$$
\begin{gathered}
\left(\frac{\partial^{2}}{\partial \tau^{2}}-\frac{\partial^{2}}{\partial x^{2}}\right) v_{4}=2 \alpha_{0} \frac{\partial^{2} v_{2}}{\partial \tau^{2}}+\alpha_{0} \frac{\partial v_{0}}{\partial \tau}-\frac{\partial v_{1}}{\partial \tau} \\
\left.\frac{\partial v_{4}}{\partial x}\right|_{x=0}=0,\left.\quad v_{4}\right|_{x=1}=-\left.\alpha_{0} \frac{\partial v_{2}}{\partial x}\right|_{x=1}-\left.\frac{1}{2} u_{1}\right|_{x=1}-\left.\frac{\alpha_{0}}{2} u_{0}\right|_{x=1},
\end{gathered}
$$

решение которой ищем в виде

$$
v_{4}=\sum_{n=1}^{\infty} z_{n} V_{n}(x) \exp \left(i \omega_{n} \tau\right)+\bar{z}_{n} \bar{V}_{n}(x) \exp \left(-i \omega_{n} \tau\right)
$$

В результате для определения коэффициентов $V_{n}, n=1,2, \ldots$, приходим к краевым задачам

$$
\begin{aligned}
V_{n}^{\prime \prime}+\omega_{n}^{2} V_{n} & =-2 \alpha_{0}(-1)^{n-1} \omega_{n}^{2} x \sin \omega_{n} x \\
V_{n}^{\prime}(0) & =0, \quad V_{n}(1)=-\alpha_{0} / 2
\end{aligned}
$$

которые оказьваются разрешимыми за счет специального выбора поправки порядка $\varepsilon^{5 / 3}$ в (6.2).

На пятом шаге из условий разрешимости в классе тригонометрических рядов вида (4.9) краевой задачи для $u_{5}, v_{5}$ получаем аналогичную (2.12) счетную систему уравнений

$$
\frac{d z_{n}}{d s}=i \alpha_{0}^{3}\left[\omega_{n}^{3} / 3-\omega_{n}\right] z_{n}+i\left(8 \omega_{n}\right)^{-1} z_{n}+f_{n}, \quad n=1,2, \ldots
$$

Привлекая, далее, функцию (4.13), убеждаемся, что эта система сворачивается в краевую задачу

$$
\frac{\partial^{2} w}{\partial s \partial y}+\frac{\alpha_{0}^{3}}{3} \frac{\partial^{4} w}{\partial y^{4}}=-\alpha_{0}^{3} \frac{\partial^{2} w}{\partial y^{2}}-\frac{w}{8}+\left[\gamma-3 w^{2}\right] \frac{\partial w}{\partial y}, \quad w(s, y+2) \equiv-w(s, y)
$$


Отметим, что, как и в предыдушем случае, задача (6.3) представляет собой лишш квазинормальную форму для исходной задачи (6.1). Однако и здесь аналог теоремы 2.1 о соответствии между их грубьми периодическими решениями остается в силе.

Выполняя в задаче (6.3) замену $y-\alpha_{0}^{3} s \rightarrow y$, подходящие нормировки переменных $s$, $y, w$ и переобозначая в конечном итоге $s$ через $t, y$ через $x$, приведем ее к каноническому виду

$$
\frac{\partial^{2} w}{\partial t \partial x}+a^{2} \frac{\partial^{4} w}{\partial x^{4}}=w+\lambda\left(1-w^{2}\right) \frac{\partial w}{\partial x}, \quad w(t, x+1) \equiv-w(t, x),
$$

где $a \neq 0, \lambda>0$. Эта краевая задача и является искомьм гибридом, включаюшим в себя задачи (2) и (3) как частные случаи. Действительно, к первой же из них приходим, полагая $a=0$ в (6.4). Для получения же задачи (3) в (6.4) положим $a^{2}=\sigma^{2} \lambda$, вьполним нормировку времени $\lambda t \rightarrow t$, сократим на $\lambda$ и устремим $\lambda$ к бесконечности. В результате приходим к задаче

$$
\frac{\partial^{2} w}{\partial t \partial x}+\sigma^{2} \frac{\partial^{4} w}{\partial x^{4}}=\left(1-w^{2}\right) \frac{\partial w}{\partial x}, \quad w(t, x+1) \equiv-w(t, x),
$$

которая после "сбрасывания" дифференцирования по $x$ приводится к виду (3).

В заключение добавим, что гибридная краевая задача (6.4) наследует свойства задачи (3), а именно, при любом фиксированном $a \neq 0$ она имеет счетное число устойчивых периодических решений (4.16) с номерами $n$, большими некоторого $n_{0}$. Тем самым, в исходной краевой задаче (6.1) при условии (6.2) и при всех $0<\varepsilon \ll 1$ по-прежнему наблюдается феномен буферности.

\section{§ 7. Заключительные замечания}

Итак, фундаментальность краевых задач (2) и (3) проиллюстрирована в очередной раз на новом фактическом материале. Ясно также, что список конкретных радиофизических примеров, анализ которых приводит к задачам (2) и (3), можно продолжить. Однако мы сознательно ограничились рассмотрение лишш круга вопросов, связанных с распределенными аналогами генератора Ван-дер-Поля. Тем самым, попутно решена интересная прикладная проблема, включающая в себя следуюшие два аспекта. Во-первых, описаны физические идеи, лежашие в основе создания указанных генераторов. Во-вторых, проведено теоретическое исследование автоколебаний в соответствующих математических моделях и выявлена главная особенность - феномен буферности.

Наконец, следует отметить, что к настоящему времени имеется целая серия экспериментов (поставленных В.Ф. Камбуловым и частично опубликованных в [20], [21]), подтверждающая физическую реализуемость этого феномена. Так, например, в генераторе, моделируюшемся краевой задачей (1.8), удалось обнаружить до шести различных сосуществующих периодических режимов, реализация каждого из которых зависела (при фиксированных прочих параметрах) только от задания подходящих начальных условий для тока и напряжения.

Возвращаясь к математическим аспектам рассмотренных проблем, напомним, что, как уже отмечалось во Введении, при исследовании конкретных гиперболических систем могут возникать не сами модельные задачи (2) и (3), а их модификации. Для того чтобы убедиться в этом, рассмотрим два типовых примера. 
В качестве первого примера возьмем краевую задачу

$$
u_{t t}+\left(3 u^{2}-\varepsilon\right) u_{t}+\mu u=u_{x x},\left.\quad u\right|_{x=0}=\left.u_{x}\right|_{x=1}=0,
$$

где $0<\varepsilon, \mu \ll 1$, и заметим, что ее собственные частоты

$$
\omega_{n}=\sqrt{\mu+\pi^{2}(2 n+1)^{2} / 4}, \quad n=0,1, \ldots,
$$

при $\mu=0$ находятся во всевозможных резонансных соотношениях нечетных порядков, а при $\mu>0$ они теряют эквидистантность. Поэтому подставим в нее $\mu=\varkappa \varepsilon$, $\varkappa=$ const $>0$, и изучим ее динамику при уменњшении $\varkappa$ методом бесконечномерной нормализации.

Следуя описанной в $§ 2$ методике, положим в (7.1)

$$
\begin{aligned}
u & =\sqrt{\varepsilon} u_{0}(t, s, x)+\varepsilon^{3 / 2} u_{1}(t, s, x)+\cdots, \quad s=\varepsilon t, \\
u_{0} & =\sum_{n=0}^{\infty}\left[z_{n}(s) \exp \left(i \omega_{n} t\right)+\bar{z}_{n}(s) \exp \left(-i \omega_{n} t\right)\right] \sin \omega_{n} x,
\end{aligned}
$$

где $\omega_{n}=\pi(2 n+1) / 2$, и приравняем коэффициенты при $\varepsilon^{3 / 2}$. В итоге из условий разрешимости в классе 4-периодических по $t$ функций краевой задачи для $u_{1}$ выводим аналогичную (2.12) счетную систему дифференциальных уравнений, которая сворачивается в скалярную краевую задачу

$$
2 \frac{\partial^{2} w}{\partial s \partial y}=-\varkappa w+\left[1-3 M\left(w^{2}\right)-3 w^{2}\right] \frac{\partial w}{\partial y}, \quad w(s, y+2) \equiv-w(s, y)
$$

Здесь $M(*)$ - среднее значение по $y$,

$$
w=\sum_{n=0}^{\infty}\left(w_{n} \exp \left(i \omega_{n} y\right)+\bar{w}_{n} \exp \left(-i \omega_{n} y\right)\right), \quad w_{n}=-i z_{n} / 2
$$

Далее, посредством подходящих нормировок $s, y, w$ и замен $y$ на $x, s$ на $t$ приведем задачу (7.2) к каноническому виду:

$$
\frac{\partial^{2} w}{\partial t \partial x}=w+\lambda\left[1-M\left(w^{2}\right)-w^{2}\right] \frac{\partial w}{\partial x}, \quad w(t, x+1) \equiv-w(t, x)
$$

где $\lambda=(2 \varkappa)^{-1}>0, M(*)$ - среднее значение по $x$. Остается добавить, что краевые задачи (2) и (7.3) имеют не только сходную структуру, но, как показано в [11], и идентичные динамические свойства по параметру $\lambda$.

Рассмотренньй пример представляет интерес и в том плане, что иллюстрирует еще одну отмеченную во Введении возможность реализации схемы (1), связанную с переходом от неэквидистантного спектра собственных частот к эквидистантному.

Однако, как показано в $\S \S 4,5$, данный переход может вызвать и прямо противоположные последствия, т.е. увеличение числа сосушествующих устойчивых циклов. 
Для того чтобы убедиться в этом еще раз, рассмотрим второй пример - краевую задачу

$$
u_{t t}+\left(3 u^{2}-\varepsilon\right) u_{t}=u_{x x}, \quad-\left.\mu u_{x}\right|_{x=0}+\left.u\right|_{x=0}=0,\left.\quad u_{x}\right|_{x=1}=0
$$

где по-прежнему $0<\varepsilon, \mu \ll 1$. Поскольку ее собственные частоты - положительные корни уравнения $\operatorname{ctg} \omega=\mu \omega$, то здесь, как и в $\S 4$, целесообразно положить

$$
\mu=\alpha \varepsilon^{1 / 3}, \quad \alpha=\text { const }>0 .
$$

Применяя, далее, к задаче (7.4) при условии (7.5) описанный в $\S 4$ метод, приходим в конечном итоге к модельной краевой задаче

$$
\frac{\partial w}{\partial t}+a^{2} \frac{\partial^{3} w}{\partial x^{3}}=w-3 M\left(w^{2}\right) w-w^{3}, \quad w(t, x+1) \equiv-w(t, x)
$$

где $a^{2}=\alpha^{3} / 12, M(*)$ - среднее значение по $x$. Отметим, наконец, что, как установлено в [11], данная задача, так же, как и задача (2), обладает свойством гипербуферности.

Суммируя все вышесказанное, приходим к выводу, что инвариантность задач (2) и (3) имеет место в некотором обобшенном смысле, т.е. с точностью до незначительных структурных изменений, не влияющих принципиально на динамику. Выбор же в качестве эталонных именно задач (2) и (3) обусловлен их максимальной структурной простотой.

В связи с этим уместно напомнить, что задача (2) впервые была получена в статье [15], а задача (3) сначала, безотносительно к каким-либо приложениям, рассматривалась в работе [10] и лишь впоследствии нашла применение в радиофизике (см. $[11],[16])$. Что же касается гибридной краевой задачи (6.4), то она является новой.

\section{СПИСОК ЛИТЕРАТУРЫ}

[1] Захаров А. А., Колесов Ю. С. Пространственно неоднородные режимы в задаче хищник-жертва // Нелинейные колебания и экология. Ярославль: ЯрГУ, 1984. С. 3-15.

[2] Колесов А. Ю. Существование асимптотически большого числа устойчивых бегущих волн в одной трехмерной системе параболических уравнений // Методы качественной теории дифференциальных уравнений. Горький: ГГУ, 1986. С. 57-66.

[3] Колесов А. Ю. Автоколебания сингулярно возмущенных параболических систем первой степени негрубости // Матем. заметки. 1991. Т. 49. № 5. С. 62-69.

[4] Колесов А.Ю. Бифуркация периодических решений сингулярно возмущенных параболических систем первой степени негрубости // Укр. матем. журн. 1990. Т. 42 . №8. C. $1037-1042$.

[5] Колесов А.Ю. Теоретическое объяснение явления диффузионной буферности // Моделирование динамики популяций. Н. Новгород: НГУ, 1990. С. 35-38.

[6] Колесов А. Ю., Розов Н.Х. Диффузионная буферность в одной математической модели биологии // Изв. РАН. Сер. матем. 1998. Т. 62. № 5. С. 135-164.

[7] Колесов А. Ю., Колесов Ю. С. Бифуркация автоколебаний сингулярно возмущенного волнового уравнения // Докл. АН СССР. 1990. Т. 315. № 2. С. 281-283.

[8] Колесов А.Ю. Устойчивость автоколебаний телеграфоного уравнения, бифурцирующих из состояния равновесия // Матем. заметки. 1992. Т. 51. № 2. С. 59-65.

[9] Колесов А. Ю., Розов Н. Х. Построение периодических решений уравнения типа Буссинеска с помощью метода квазинормальных форм // Фунд. и прикл. матем. 1995. Т. 1. № 1 . C. $207-220$. 
[10] Колесов А. Ю. Существование счетного числа устойчивых циклов в средах с дисперсией // Изв. РАН. Сер. матем. 1995. Т. 59. № 3. С. 141-158.

[11] Колесов А. Ю., Мищенко Е. Ф., Розов Н. Х. Асимптотические методы исследования периодических решений нелинейных гиперболических уравнений // Тр. МИАН. 1998. T. 222. C. 1-192.

[12] Витт А.А. Распределенные автоколебательные системы // Журн. технич. физ. 1934. T. 4. № 1. C. 144-157.

[13] Витт А.А. К теории скрипичной струны // Журн. технич. физ. 1936. Т. 6.№9. C. $1459-1479$.

[14] Азьян Ю. М., Мигулин В. В. Об автоколебаниях в системах с запаздывающей обратной связью // Радиотехн. и электроника. 1956. Т. 1. №1. С. 126-130.

[15] Камбулов В.Ф., Колесов А. Ю. Об одном модельном гиперболическом уравнении, возникающем в радиофизике // Матем. моделирование. 1996. Т. 8. № 1. С. 93-102.

[16] Камбулов В.Ф., Колесов А. Ю., Розов Н. Х. Теоретический и экспериментальный анализ феномена буферности в длинной линии с туннельным диодом // Дифференц. уравнения. 1997. Т. 33. №5. С. $638-645$.

[17] Колесов Ю.С. Бифуркация инвариантных торов параболических систем с малой диффузией // Матем. сб. 1993. Т. 184. № 3. С. 121-136.

[18] Колесов Ю.С.Асимптотика и устойчивость нелинейных параметрических колебаний сингулярно возмущенного телеграфиного уравнения // Матем. сб. 1995. Т. 186. № 10. C. $57-72$.

[19] Камбулов В.Ф. Резонансность как источник релаксационных колебаний в системах телеграфных уравнений // Докл. АН. 1994. Т. 334. № 5. С. 569-570.

[20] Камбулов В.Ф. Модель распределенного автогенератора Ван-дер-Поля // Радиотехн. и электроника. 1997. Т. 42. № 9. С. 1121-1124.

[21] Камбулов В.Ф., Колесов А. Ю., Розов Н. Х. Исследование автоколебаний в генераторе Ван-дер-Поля с распределенными параметрами // Вестник МГУ. Сер. 3. Физика. Астрономия. 1999. № 3. С. 23-27.

[22] Андронов А. А., Витт А. А., Хайкин С. Э. Теория колебаний. М.: Физматгиз, 1959.

[23] Боголюбов Н. Н., Митропольский Ю. А. Асимптотические методы в теории нелинейных колебаний. М.: Наука, 1974.

[24] Митропольский Ю. А., Лыкова О. В. Интегральные многообразия в нелинейной механике. М.: Наука, 1973.

[25] Хартман П.Ф. Обыкновенные дифференциальные уравнения. М.: Мир, 1970.

[26] Ланда П. С. Нелинейные колебания и волны. М.: Наука, 1997.

[27] Колесов А. Ю. Релаксационные циклы нелинейного волнового уравнения, гладко зависящего от параметров // Докл. АН. 1995. Т. 341. № 2. С. 158-160.

[28] Камбулов В.Ф., Колесов А. Ю., Розов Н. Х. Бифуркация пространственно неоднородных циклов у нелинейного волнового уравнения с малой диффузией // Труды ММО. 1997. Т. 59. С. 197-220.

[29] Колесов Ю.С., Майоров В.В. Новый метод исследования устойчивости решений линейных дифференциальных уравнений с близкими к постоянным почти периодическими коэффициентами // Дифференц. уравнения. 1974. Т. 10. №10. С. 1778-1788.

Ярославский государственный университет им. П.Г. Демидова;

Поступила в редакцию Математический институт им. В. А. Стеклова РАН; 05.01 .2000

Московский государственный университет им. М. В. Ломоносова 